\title{
Pacific
}

Journal of

Mathematics

\section{ON SOME EXPLICIT FORMULAS IN THE THEORY OF WEIL REPRESENTATION}

\author{
R. RANGA RAO
}




\title{
ON SOME EXPLICIT FORMULAS IN THE THEORY OF WEIL REPRESENTATION
}

\author{
R. RANGA RAO
}

The object of this paper is to derive some explicit formulae concerning the Weil representation that allow us to define this projective representation in a unique manner for each choice of symplectic basis.

Let $F$ be a self-dual locally compact field of char $\neq 2$ and $X$ a symplectic vector space over $F$. Let $V, V^{*}$ be two transversal Lagrangian subspaces. Then a classical construction due to Shale-SegalWeil gives a projective representation of the symplectic group $\operatorname{Sp}(X)$ in the Schwartz-space of $V$. The operators $\xi(\sigma)$ corresponding to each $\sigma \in \operatorname{Sp}(X)$ are determined uniquely only up to a scalar multiple. The starting point of this paper is an explicit integral formula for these operators $\xi(\sigma)$, valid for all $\sigma \in \operatorname{Sp}(X)$. In fact (see Lemma 3.2) we have for each $\sigma \in \operatorname{Sp}(X)$

$$
\xi(\sigma) \varphi: x \rightarrow \int_{V^{*} / \operatorname{ker} \gamma} f_{\sigma}\left(x, x^{*}\right) \varphi\left(x \alpha+x^{*} \gamma\right) d \mu_{\sigma}
$$

where $\mu_{\sigma}$ is a Haar measure on $V^{*} / \operatorname{ker} \gamma$, and $f_{\sigma}$ is the character of second degree on $X$, associated to $\sigma$. Here $\sigma=\left[\begin{array}{ll}\alpha & \beta \\ \gamma & \delta\end{array}\right]$ is the matrix representation of $\sigma$ in the decomposition $X=V+V^{*}$. This formula is known and already present in Weil's paper when $\gamma=0$ or when $\gamma$ is an isomorphism. The extension of its validity for all $\sigma$ enables us to show that it is possible to define the projective representation in a unique way for each choice of symplectic basis. Let $e_{1}, \ldots, e_{n}, e_{1}^{*}, \ldots, e_{n}^{*}$ be a symplectic basis of $X$ such that $e_{1}, \ldots, e_{n}\left(e_{1}^{*}, \ldots, e_{n}^{*}\right)$ is a basis of $V\left(V^{*}\right)$. Let $W$ be the finite subgroup of $\operatorname{Sp}(X)$ consisting of all $\sigma$ such that $\left\{e_{i}, e_{i}^{*}\right\} \sigma \subseteq\left\{ \pm e_{i}, \pm e_{i}^{*}\right\}$ for each $i$. Then one has the well-known Bruhat decomposition $\operatorname{Sp}(X)=P W P$, where $P$ is the stabilizer of $V^{*}$. Then it is shown that it is possible to make consistent choices of the Haar measures $\mu_{\sigma}$ so that (1) $\xi\left(p_{1} \sigma p_{2}\right)=\xi\left(p_{1}\right) \xi(\sigma) \xi\left(p_{2}\right)$ for all $p_{1}, p_{2} \in P$ and $(2) \xi\left(\sigma_{1} \sigma_{2}\right)=\xi\left(\sigma_{1}\right) \xi\left(\sigma_{2}\right)$ for all $\sigma_{1}, \sigma_{2} \in W$. Moreover all such are determined. Among these there is one choice $\sigma \rightarrow r(\sigma)$ called the standard model which in addition satisfies nonnegativity properties similar to those of the Fourier Transform. All 
this is done in $\S 3$ and the main result is stated in Theorem 3.6. For the standard model the multiplier or the 2-cocycle $c\left(\sigma_{1}, \sigma_{2}\right)$ is explicitly described in $\S 4$, Theorem 4 in terms of the Leray invariant, i.e. $c\left(\sigma_{1}, \sigma_{2}\right)$ is the Weil index of the Leray invariant of the Lagrangian subspaces $V^{*}, V^{*} \sigma_{2}^{-1}, V^{*} \sigma_{1}$. This formula generalizes one given by Weil when $\sigma_{1}, \sigma_{2}, \sigma_{1} \sigma_{2}$ belongs to the big Bruhat cell. Finally we find normalizing constants $m(\sigma)$ such that $r^{\sim}(\sigma)=m(\sigma) r(\sigma)$ is metaplectic, i.e. the associated multiplier is \pm 1 valued. An explicit formula for the multiplier $c^{\sim}\left(\sigma_{1}, \sigma_{2}\right)$ of $r$ is given in Theorem 5.1. This generalizes a result of Kubota. In fact the formula given reduces to that of Kubota when $\operatorname{dim} X=2$. Although Moore [18] has described the cohomology groups, the explicit formula for the multiplier given here appears to be new.

Preliminaries on symplectic geometry are discussed in $\S 2$. Here the Leray invariant ( $=$ an isometry class of a certain inner product space) of an ordered triplet of Lagrangian subspaces is defined. The main result here is Theorem 1 which shows that the $\operatorname{Sp}(X)$-orbits of an ordered triplet of Lagrangian subspaces are parametrized by the various dimensions and the Leray invariant. Theorem 2 gives an application of this to the structure of $\operatorname{Sp}(X)$. This theorem is used in the computation of the multipliers. Weil in his paper [16], introduced a constant $\gamma(f)$ for each nondegenerate character of second degree. We call this constant the Weil index of $f$. The definition and properties of the Weil index, its relation to the Hilbert and Hasse symbols and various known computations of the Weil index are collected together in the appendix.

There is a large literature on the subject. For further references see the recent books of Guillemin and Sternberg [6] and Wallach [17] for the real case and Gelbart [4] for $\mathrm{SL}_{2}$ and $p$-adic case and Gerardin [5] for the case of finite fields. Part of the results of the paper were obtained when the author was visiting Tata Institute, Bombay, the Indian Statistical Institute, New Delhi, and the Forschungsinstitut of E. T. H., Zurich during 1977-78. The author would like to express his thanks for their hospitality and support. The author is also indebted to C. Moreno for many stimulating conversations.

Postscript: This paper was written in 1978 and widely circulated at that time. For various reasons it has not been published. Since this paper is referred to in many published papers, it was felt that its publication is still desirable. No attempt has been made to update the references since they are too numerous. Instead we refer the reader 
to the books of G. Folland [19] and G. Lion and M. Vergne, [20] and also [21].

\section{Preliminaries on symplectic geometry.}

2.1. Let $k$ be a field and $X$ a symplectic vector space over $k$, i.e. $X$ is a finite dimensional $k$-vector space with a non-degenerate bilinear form $x, y \rightarrow\langle x, y\rangle$, which is symplectic, or $\langle x, x\rangle=0$ for all $x \in X$. If $X$ and $Y$ are symplectic vector spaces, a $k$-linear isomorphism of $X$ and $Y$ which preserves the symplectic structures is called a symplectomorphism. Let $\operatorname{Sp}(X)$ denote the group of symplectomorphisms of $X$ onto itself. A subspace $L$ of $X$ is said to be isotropic if $L \cap L^{\perp} \neq\{0\}$, nonisotropic if $L \cap L^{\perp}=\{0\}$. A nonisotropic subspace is also called a symplectic subspace, since the restriction of the symplectic form to it is nondegenerate. If $X_{1} \subset X$ is a symplectic subspace, so is $X_{2}=X_{1}^{\perp}$ and $X=X_{1}+X_{2}$ direct sum. A subspace $L$ is totally isotropic if $L \subset L^{\perp}$ and maximal totally isotropic or $L a$ grangian if $L=L^{\perp}$. Two subspaces $L_{1}, L_{2}$ are said to be transversal if $L_{1} \cap L_{2}=\{0\}$. An ordered basis $\left\{v_{1}, \ldots, v_{2 n}\right\}$ of $X$ is said to be a symplectic basis if the following relations hold:

$$
\left\langle v_{i}, v_{j}\right\rangle=\left\langle v_{i+n}, v_{j+n}\right\rangle=0 ; \quad\left\langle v_{i}, v_{j+n}\right\rangle=\delta_{i j}
$$

for all $i, j$ with $1 \leq i, j \leq n$. The following lemma and its consequence will repeatedly be used in this section (for proof see Bourbaki [1]).

LEMMA 2.1. (i) Let $v_{1}, \ldots, v_{m}$ be linearly independent vectors of $X$, satisfying $\left\langle v_{i}, v_{j}\right\rangle=0$ for all $i, j$. Then there exist vectors $w_{1}, \ldots$, $w_{m}$ in $X$ such that

$$
\left\langle w_{i}, w_{j}\right\rangle=0 \text { for all } i, j \text { and }\left\langle v_{i}, w_{j}\right\rangle=\delta_{i j} .
$$

(ii) If $L$ is a Lagrangian subspace, then $\operatorname{dim} X=2 \operatorname{dim} L$ and there exists a Lagrangian subspace transversal to $L$.

(iii) If $L_{1}, L_{2}$ are two transversal Lagrangian subspaces, and $v_{1}, \ldots, v_{n}$ is a basis of $L_{1}$, there exists a basis $v_{n+1}, \ldots, v_{2 n}$ of $L_{2}$ such that $v_{1}, \ldots, v_{2 n}$ is a symplectic basis of $X$.

(iv) Let $X$ and $Y$ be symplectic vector spaces and $L_{1}, L_{2}\left(M_{1}, M_{2}\right)$ be Lagrangian subspaces of $X$ (of $Y$ ). If $\operatorname{dim} X=\operatorname{dim} Y$, and $\sigma$ is an arbitrary $k$-linear map of $L_{1}$ onto $M_{1}$ then $\sigma$ can be extended as a symplectomorphism of $X$ onto $Y$, mapping $L_{i}$ on $M_{i}, i=1,2$. 
2.2. Following Weil, we write the action of the group $\operatorname{Sp}(X)$ on $X$ on the right. Let $\Lambda(X)$ denote the set of all Lagrangian subspaces of $X$. It is clear that $\operatorname{Sp}(X)$ acts transitively on $\Lambda(X)$. For $L \in \Lambda(X)$, let $P_{L}$ denote the isotropy subgroup at $L$ for the action of $\operatorname{Sp}(X)$, i.e.

$$
P_{L}=\{\sigma \in \operatorname{Sp}(X) \mid L \cdot \sigma=L\}
$$

Also write

$$
N_{L}=\{\sigma \in \operatorname{Sp}(X) \mid v \sigma=v \text { for all } v \in L\} .
$$

In the rest of the section we develop some results (needed later) on the orbits of $P_{L}$ on $\Lambda(X)$ and on $\Lambda(X) \times \Lambda(X)$.

LemMA 2.2. Let $L_{1}, L_{2} \in \Lambda(X)$. Then there exists a decomposition $X=X_{1}+X_{2}$ into a direct sum of orthogonal symplectic subspaces $X_{1}$, $X_{2}$ such that

(1) $L_{1}, L_{2}$ commute with the decomposition, i.e. $L_{i}=L_{i} \cap X_{1}+$ $L_{i} \cap X_{2}, i=1,2$ and $L_{i} \cap X_{j}$ are Lagrangian subspaces of $X_{j}$.

(2) On $X_{1}, L_{1}=L_{2}$, i.e. $L_{1} \cap X_{1}=L_{2} \cap X_{1}$.

(3) On $X_{2}, L_{1}, L_{2}$ are transversal, i.e. $\left(L_{1} \cap X_{2}\right) \cap\left(L_{2} \cap X_{2}\right)=\{0\}$.

Proof. Let $M=L_{1} \cap L_{2}$. Then $M$ is a totally isotropic subspace. From Lemma 1, it follows that there exists a totally isotropic subspace $F$, such that $X=M^{\perp}+F$ direct sum. Let $X_{1}=M+F, X_{2}=X_{1}^{\perp}$. Then $X_{1}, X_{2}$ are symplectic subspaces. Now $M \subset M^{\perp}$, so we have $M^{\perp} \cap X_{1}=M$, and $M^{\perp}=M+X_{2}$. Since $M \subset L_{i} \subset M^{\perp}$ it follows that $L_{i}=M+L_{i} \cap X_{2}=L_{i} \cap X_{1}+L_{i} \cap X_{2}$. From dimension considerations it follows that $L_{i} \cap X_{j}$ are Lagrangian subspaces of $X_{j}$. It is clear that $L_{1} \cap X_{1}=M=L_{2} \cap X_{1}$. This clearly implies (2) and (3).

Lemma 2.3. Let $L_{1}, L_{2}, L \in \Lambda(X)$ be arbitrary. Then

(1) there exists $\sigma \in N_{L}$ such that $L_{1} \sigma=L_{2}$ if and only if $L_{1} \cap L=$ $L_{2} \cap L$. The element $\sigma$ is unique if $L_{1}$ and $L_{2}$ are both transversal to $L$.

(2) There exists $\sigma \in P_{L}$ such that $L_{1} \sigma=L_{2}$ if and only if $\operatorname{dim} L_{1} \cap$ $L=\operatorname{dim} L_{2} \cap L$.

Proof. (1) Suppose $L_{1} \cap L=L_{2} \cap L=M$. Choose an isotropic subspace $F$ such that $M^{\perp}+F=X$ direct sum. Let $X_{1}=M+F$ and $X_{2}=X_{1}^{\perp}$. Then the decomposition $X=X_{1}+X_{2}$ has all the 
properties of Lemma 2.2, for both the pairs $L_{1}, L$ and $L_{2}, L$. Thus on $X_{1}, L_{1}=L_{2}=L$ and on $X_{2}, L_{1}$ and $L_{2}$ are both transversal to $L$. Let $\sigma=\operatorname{diag}\left(\sigma_{1}, \sigma_{2}\right)$, where $\sigma_{1}$ is the identity element of $\operatorname{Sp}\left(X_{1}\right)$ and $\sigma_{2}$ is any element of $\operatorname{Sp}\left(X_{2}\right)$ such that $\left(L_{1} \cap X_{2}\right) \sigma_{2}=L_{2} \cap X_{2}$ and $\sigma_{2}=$ identity on $L \cap X_{2}$. The existence of such a $\sigma_{2}$ can be seen by considering symplectic bases associated to the decompositions $X_{2}=L \cap X_{2}+L_{1} \cap X_{2}=L \cap X_{2}+L_{2} \cap X_{2}$. It is then clear that $\sigma \in N_{L}$ and $L_{1} \sigma=L_{2}$. It remains to check uniqueness when $L_{1}, L_{2}$ are both transversal to $L$. Suppose $L_{1} \sigma=L_{2}=L_{1} \sigma^{\prime}$. Let $u=\sigma \sigma^{\prime-1}$. Then $u \in N_{L}$ and $L_{1} u=L_{1}$. If $x \in L_{1}$ and $y \in L$, then

$$
\langle x u-x, y\rangle=\langle x \cdot u, y\rangle-\langle x, y\rangle=\langle x u, y u\rangle-\langle x, y\rangle=0 .
$$

Thus $x u-x \in L^{\perp}=L$. But $x u-x \in L_{1}$. Thus $x \cdot u=x$ and $u=\mathrm{id}$ on $L_{1}$ also. Since $L_{1}+L=X$, it follows that $u=\mathrm{id}$.

Proof of (2). Let $X=X_{1}+X_{2}=Y_{1}+Y_{2}$ be the decompositions of Lemma 2 for the pairs $L_{1}, L$ and $L_{2}, L$ respectively. Now $\operatorname{dim} X_{1}=2 \operatorname{dim} L \cap L_{1}=2 \operatorname{dim} L \cap L_{2}=\operatorname{dim} Y_{1}$. Thus there exists a symplectomorphism $\sigma_{1}$ of $X_{1}$ into $Y_{1}$ which takes $L \cap X_{1}$ to $L \cap Y_{1}$. Let $\sigma_{2}$ be a symplectomorphism of $X_{2}$ onto $Y_{2}$ which takes $L \cap X_{2}$ to $L \cap Y_{2}$. If $\sigma=\operatorname{diag}\left(\sigma_{1}, \sigma_{2}\right)$, then $\sigma \in P_{L}$ and $L_{1} \sigma \cap L=L_{2} \cap L$. The result now follows from the first part.

2.3. Let $L_{1}, L_{2}, L_{3}$ be Lagrangian subspaces which are pairwise transversal. Then by Lemma 3 there exists a unique $u \in N_{L_{1}}$ such that $L_{2} \cdot u=L_{3}$. Let $u$ be represented by the matrix

$$
u=\left[\begin{array}{ll}
I & \rho \\
0 & I
\end{array}\right]
$$

in the decomposition $X=L_{2}+L_{1}$. Since $u$ is symplectic one has that, for $x, y \in L_{2}$,

$$
Q(x, y)=\langle x, y \cdot u\rangle=\langle x, y \cdot \rho\rangle=\langle y, x \cdot \rho\rangle
$$

is a symmetric bilinear form on $L_{2}$. Since $L_{3}=\left\{x+x \cdot \rho: x \in L_{2}\right\}$, the transversality of $L_{2}$ and $L_{3}$ implies that $\rho$ is injective or the symmetric bilinear form is nondegenerate.

DEFINITION 2.4. For any three pairwise transversal Lagrangian subspaces $L_{1}, L_{2}, L_{3}$ of $X$, let $q\left(L_{1}, L_{2}, L_{3}\right)$ denote the isometry class of the inner product space $\left\{L_{2}, Q(\cdot, \cdot)\right\}$ introduced above. We call $q\left(L_{1}, L_{2}, L_{3}\right)$ the Leray invariant of $L_{1}, L_{2}, L_{3}$ (see Leray [8]). 
LEMMA 2.5. Let $X$ and $Y$ be symplectic vector spaces of the same dimension. Let $L_{1}, L_{2}, L_{3} \in \Lambda(X), L_{1}^{\prime}, L_{2}^{\prime}, L_{3}^{\prime} \in \Lambda(Y)$ be two triplets of pairwise transversal Lagrangian subspaces. Then there exists a symplectomorphism $\sigma$ of $X$ onto $Y$ such that

$$
L_{j} \cdot \sigma=L_{j}^{\prime}, \quad j=1,2,3
$$

if and only if

$$
q\left(L_{1}, L_{2}, L_{3}\right)=q\left(L_{1}^{\prime}, L_{2}^{\prime}, L_{3}^{\prime}\right)
$$

Proof. Suppose first $L_{j} \sigma=L_{j}^{\prime}, j=1,2,3$, for a symplectomorphism $\sigma$. Let $u \in N_{L_{1}}$ be such that $L_{2} \cdot u=L_{3}$. Let $u^{\prime}=\sigma^{-1} u \sigma$. Then it is clear that $u^{\prime} \in N_{L_{1}} \cdot \sigma=N_{L_{1}^{\prime}}$ and $L_{2}^{\prime} \cdot u^{\prime}=L_{2} \cdot \sigma u^{\prime}=L_{2} u \sigma=$ $L_{3} \sigma=L_{3}^{\prime}$. Thus, for $x, y \in L_{2}$

$$
Q^{\prime}(x \cdot \sigma, y \cdot \sigma)=\langle x \cdot \sigma, y \cdot \sigma u\rangle=\left\langle x, y \cdot u^{\prime}\right\rangle=Q(x, y) .
$$

Thus $Q$ and $Q^{\prime}$ are isometric. Conversely suppose $q\left(L_{1}, L_{2}, L_{3}\right)=$ $q\left(L_{1}^{\prime}, L_{2}^{\prime}, L_{3}^{\prime}\right)$. Let $u \in N_{L_{1}}, u^{\prime} \in N_{L_{1}^{\prime}}$ be the unique elements such that

$$
L_{2} \cdot u=L_{3}, \quad L_{2}^{\prime} \cdot u^{\prime}=L_{3}^{\prime} .
$$

Let $Q^{\prime}\left(x^{\prime}, y^{\prime}\right)=\left\langle x^{\prime}, y^{\prime} \cdot u^{\prime}\right\rangle, x^{\prime}, y^{\prime} \in L_{2}^{\prime}$. Similarly $Q(x, y)=$ $\langle x, y \cdot u\rangle, x, y \in L_{2}$. Since $Q$ and $Q^{\prime}$ are isometric, there exists a $k$-linear isomorphism $\sigma$ of $L_{2}$ onto $L_{2}^{\prime}$ such that $Q^{\prime}(x \cdot \sigma, y \cdot \sigma)=$ $Q(x, y)$ for all $x, y$. Now $X=L_{1}+L_{2}$ and $Y=L_{1}^{\prime}+L_{2}^{\prime}$. By using symplectic bases, it is clear $\sigma$ can be extended to a symplectomorphism of $X$ onto $Y$ such that $L_{1} \cdot \sigma=L_{1}^{\prime}$. Let $L_{3}^{\prime \prime}=L_{3} \cdot \sigma$. We have only to show that $L_{3}^{\prime \prime}=L_{3}^{\prime}$. Now $L_{3}^{\prime \prime}=L_{2} u \cdot \sigma=L_{2} \sigma\left(\sigma^{-1} u \sigma\right)=$ $L_{2}^{\prime} \cdot u^{\prime \prime}$ where $u^{\prime \prime}=\sigma^{-1} u \sigma \in N_{L_{1}^{\prime}}$. Now, for all $x, y \in L_{2}$,

$$
\langle x, y u\rangle=Q(x, y)=Q^{\prime}(x \sigma, y \sigma)=\left\langle x \cdot \sigma, y \cdot \sigma \cdot u^{\prime}\right\rangle .
$$

On the other hand

$$
\left\langle x \cdot \sigma, y \cdot \sigma \cdot u^{\prime \prime}\right\rangle=\langle x \cdot \sigma, y \cdot u \sigma\rangle=\langle x, y u\rangle .
$$

Thus $\left\langle x^{\prime}, y^{\prime} \cdot u^{\prime \prime}\right\rangle=\left\langle x^{\prime}, y^{\prime} \cdot u^{\prime}\right\rangle$ for all $x^{\prime}=x \cdot \sigma, y^{\prime}=y \cdot \sigma \in L_{2}^{\prime}$. Since both $u^{\prime}, u^{\prime \prime} \in N_{L_{1}^{\prime}}$, it follows that $u^{\prime}=u^{\prime \prime}$. But $L_{2}^{\prime} \cdot u^{\prime}=L_{3}^{\prime}$. Thus $L_{3}^{\prime}=L_{3}^{\prime \prime}=L_{3} \cdot \sigma$.

For the sake of clarifying the nature of the invariant $q\left(L_{1}, L_{2}, L_{3}\right)$ we note the following. 
LEMMA 2.6. If $L_{1}, L_{2}, L_{3}$ is any triplet of pairwise transversal Lagrangian subspaces, then

$$
q\left(L_{1}, L_{2}, L_{3}\right)=q\left(L_{2}, L_{3}, L_{1}\right)=q\left(L_{3}, L_{1}, L_{2}\right)
$$

and

$$
q\left(L_{1}, L_{2}, L_{3}\right)=-q\left(L_{1}, L_{3}, L_{2}\right) .
$$

Here $-q$ is the isometry class of the symmetric bilinear form $(-Q)$, if $q$ is the isometry class of $Q$.

Proof. Let $u_{j} \in N_{L_{j}}, j=1,2,3$, be defined by $L_{2} \cdot u_{1}=L_{3}$, $L_{3} \cdot u_{2}=L_{1}, L_{1} \cdot u_{3}=L_{2}$. In the decomposition $X=L_{2}+L_{1}$, let the matrix of $u_{1}$ be

$$
u_{1}=\left[\begin{array}{ll}
I & \rho \\
0 & I
\end{array}\right] \text {. }
$$

Then $L_{3}=\left\{x_{2}+x_{2} \cdot \rho: x_{2} \in L_{2}\right\}$. Then it may be checked that

$$
u_{2}=\left[\begin{array}{cc}
I & 0 \\
-\rho^{-1} & I
\end{array}\right], \quad u_{3}=\left[\begin{array}{cc}
2 I & \rho \\
-\rho^{-1} & 0
\end{array}\right] \text {. }
$$

From this it follows that $u_{3} u_{1} u_{2}=-\left(u_{1}\right)^{-3}$. In particular $u_{3} u_{1} u_{2} \in$ $N_{L_{1}}$. Similarly $u_{1} u_{2} u_{3}=-\left(u_{2}\right)^{-3} \in N_{L_{2}}$ etc. Now $q\left(L_{1}, L_{2}, L_{3}\right)$ is the isometry class of $Q_{2}\left(x_{2}, y_{2}\right)=\left\langle x_{2}, y_{2} \cdot u_{1}\right\rangle, x_{2}, y_{2} \in L_{2}$. Note that $L_{1} \cdot u_{3}=L_{2}$ and

$$
\begin{aligned}
Q_{2}\left(x_{1} \cdot u_{3}, y_{1} \cdot u_{3}\right) & =\left\langle x_{1} \cdot u_{3}, y_{1} \cdot u_{3} u_{1}\right\rangle=\left\langle x_{1} \cdot u_{3} u_{2}, y_{1} \cdot u_{3} u_{1} u_{2}\right\rangle \\
& =\left\langle x_{1} \cdot u_{3},-y_{1}\right\rangle=\left\langle y_{1}, x_{1} \cdot u_{3}\right\rangle .
\end{aligned}
$$

Here in the last step we have used that $\left(-u_{3} u_{1} u_{2}\right) \in N_{L_{1}}$, and $u_{2} \in N_{L_{2}}$. Since $q\left(L_{3}, L_{1}, L_{2}\right)$ is the isometry class of the form $\left\{\left\langle x_{1}, y_{1} \cdot u_{3}\right\rangle, x_{1}, y_{1} \in L_{1}\right\}$, it follows that $q\left(L_{3}, L_{1}, L_{2}\right)=$ $q\left(L_{1}, L_{2}, L_{3}\right)$. The other relations are proved similarly.

REMARK. When $k$ is the field of real numbers Leray in [8] introduced the inertia of three pairwise transversal Lagrangian subspaces $L_{1}, L_{2}, L_{3}$ as follows: Consider $x_{j} \in L_{j}$ such that $x_{1}+x_{2}+x_{3}=0$. Note given one of the $x_{i}$ 's, the other are uniquely determined, thus given $x_{1}, x_{2}$ is the unique element of $L_{2}$ such that $x_{1}+x_{2} \in L_{3}$ etc. Let $\rho$ be the map $L_{2} \rightarrow L_{1}$ defined by $x_{2} \rightarrow x_{1}$, when $x_{1}+x_{2} \in L_{3}$ etc. Then $\rho$ is symmetric and it is clear that $L_{3}=\left\{x_{2}+x_{2} \rho: x_{2} \in L\right\}$. Thus the $u_{1} \in N_{L_{1}}$ such that $L_{2} \cdot u_{1}=L_{3}$ introduced earlier is given by

$$
u_{1}=\left[\begin{array}{ll}
1 & \rho \\
0 & 1
\end{array}\right]
$$


etc. The index of inertia (or the number of negative eigenvalues of $\rho$ ) is called by Leray the inertia of the triplet. Since the index of inertia of a real quadratic form of a given degree, determines its isometry class, it seems appropriate to call this the Leray invariant (see also the book [6]).

2.4. We have just seen that the isometry class $q\left(L_{1}, L_{2}, L_{3}\right)$ describes the $\operatorname{Sp}(X)$ orbit of the triple $L_{1}, L_{2}, L_{3}$, when they are pairwise transversal. Our next object is to classify the general orbit. This requires some preparation.

Lemma 2.7. Let $L_{1}, L_{2}, L_{3} \in \Lambda(X)$. Then for the pair $L_{1}, L_{2}$, there exists a decomposition $X=X_{1}+X_{2}$ with properties stated in Lemma 2, such that $L_{3}$ also commutes with the decomposition, i.e. $L_{3}=L_{3} \cap X_{1}+L_{3} \cap X_{2}$.

Proof. First consider the case when $L_{1} \cap L_{2} \cap L_{3}=\{0\}$. Then $L_{1}+L_{2}+L_{3}=X$. Thus there exists a subspace $F \subset L_{3}$ such that $X=\left(L_{1} \cap L_{2}\right)^{\perp}+F$ direct sum. If $M=L_{1} \cap L_{2}$, let $X_{1}=M+F$, $X_{2}=X_{1}^{\perp}$; then the decomposition $X=X_{1}+X_{2}$ has the required properties. It is only necessary to check that $L_{3}$ commutes with the decomposition. In fact $F^{\perp}=F+X_{2}$ and since $F \subset L_{3} \subset F^{\perp}$, it follows that $L_{3}=F+L_{3} \cap X_{2}=L_{3} \cap X_{1}+L_{3} \cap X_{2}$. Now consider the general case. Let $M_{0}=L_{1} \cap L_{2} \cap L_{3}$. Let $F_{0}$ be a totally isotropic subspace such that $M_{0}^{\perp}+F_{0}=X$. Let $Y_{1}=M_{0}+F_{0}, Y_{2}=Y_{1}^{\perp}$. Then it is easy to see that $L_{1}, L_{2}, L_{3}$ commute with the decomposition $X=Y_{1}+Y_{2}$. On $Y_{1}, L_{1}=L_{2}=L_{3}$ so the lemma is valid. On $Y_{2}$, $L_{1} \cap L_{2} \cap L_{3}=\{0\}$ so that the first case discussed above applies. This completes the proof.

LemMA 2.8. Let $L_{1}, L_{2}, L_{3} \in \Lambda(X)$. Then there exist pairwise orthogonal symplectic subspaces $X_{j}, j=0,1, \ldots, 4$, such that the following hold:

(1) $X=X_{0}+\cdots+X_{4}$.

(2) $L_{i}(i=1,2,3)$ commute with the decomposition, i.e. $L_{i}=$ $\sum_{j} L_{i} \cap X_{j}$.

(3) On $X_{0}, L_{1}=L_{2}=L_{3}$.

(4) On $X_{1}, L_{2}=L_{3}$ and $L_{1}, L_{2}$ are transversal.

(5) $O n X_{2}, L_{3}=L_{1}$ and $L_{2}, L_{3}$ are transversal.

(6) $O n X_{3}, L_{1}=L_{2}$ and $L_{3}, L_{1}$ are transversal.

(7) On $X_{4}, L_{1}, L_{2}, L_{3}$ are pairwise transversal. 
Proof. Note first that the statement (2) above implies that $L_{i} \cap X_{j}$ is a Lagrangian subspace of $X_{j}$ and a property is said to hold for $L_{i}$ 's on $X_{j}$, if the same holds for $L_{i} \cap X_{j}$. Now to prove the lemma, let $X=Y_{1}+Y_{2}$ be the decomposition of the previous Lemma 5. Then $L_{1}, L_{2}, L_{3}$ commute with the decomposition and on $Y_{1}, L_{1}=L_{2}$ and on $Y_{2}, L_{1} \cap L_{3}=\{0\}$. On $Y_{1}$, apply Lemma 5 again to the triple $L_{2}, L_{3}, L_{1}$. Then $Y_{1}=X_{0}+X_{3}$, and on $X_{0}, L_{2}=L_{3}$ and on $X_{3}, L_{2}$ and $L_{3}$ are transversal. But on $Y_{1}, L_{1}=L_{2}$. Thus on $X_{0}$, $L_{1}=L_{2}=L_{3}$ and on $X_{3}, L_{1}=L_{2}$ and $L_{2}$ and $L_{3}$ are transversal. Proceeding in the same way and applying Lemma 5 to $Y_{2}$ repeatedly we get the result.

Our next object is to show that the isometry class

$$
q\left(L_{1} \cap X_{4}, L_{2} \cap X_{4}, L_{3} \cap X_{4}\right)
$$

is invariantly defined. For this we recall the technique of passing to the quotient $X_{M}$ (see Leray [8], or Guillemin and Sternberg [6]). Let $M$ be a totally isotropic subspace of $X$ and let $X_{M}=M^{\perp} / M$. Then $X_{M}$ becomes a symplectic vector space with the symplectic form defined as

$$
\langle x+M, y+M\rangle=\langle x, y\rangle, \quad x, y \in M^{\perp} .
$$

For any $L \in \Lambda(X)$, let the subspace $L_{M}$ of $X_{M}$ be defined as $L_{M}=\left(L \cap M^{\perp}\right) / M . \quad L_{M}$ is clearly a totally isotropic subspace of $X_{M}$. By dimension considerations one verifies that $L_{M}$ is actually a Lagrangian subspace of $X_{M}$ (see one of the above cited references).

Lemma 2.9. Let $L_{1}, L_{2}, L_{3} \in \Lambda(X)$. Let

$$
M=\left(L_{1} \cap L_{2}\right)+\left(L_{2} \cap L_{3}\right)+\left(L_{3} \cap L_{1}\right) .
$$

Them $M$ is a totally isotropic subspace and moreover the images $\left(L_{i}\right)_{M}$ in $X_{M}$ are pairwise transversal. Moreover

$$
q\left(\left(L_{1}\right)_{M},\left(L_{2}\right)_{M},\left(L_{3}\right)_{M}\right)=q\left(L_{1} \cap X_{4}, L_{2} \cap X_{4}, L_{3} \cap X_{4}\right)
$$

where the symplectic subspace $X_{4}$ is the one introduced in Lemma 2.8.

Proof. Since $M^{\perp}=\left(L_{1}+L_{2}\right) \cap\left(L_{2}+L_{3}\right) \cap\left(L_{3}+L_{1}\right)$, it is clear that $M \subset M^{\perp}$ or $M$ is totally isotropic. Using Lemma 6 and the notation there, it is clear that $M$ and $M^{\perp}$ both commute with the decomposition $X=\sum X_{j}$ and $M^{\perp}=M+X_{4}, M \cap X_{4}=\{0\}$. Thus the symplectic vector space $X_{4}$ is isomorphic to $X_{M}$ and the isomorphism takes $L_{i} \cap X_{4}$ onto $\left(L_{i}\right)_{M}, i=1,2,3$. The lemma follows from this and Lemma 4. 
The above lemma enables us to introduce the following definition.

Definition 2.10. For any three Lagrangian subspaces $L_{1}, L_{2}, L_{3}$, we define

$$
q\left(L_{1}, L_{2}, L_{3}\right)=q\left(\left(L_{1}\right)_{M},\left(L_{2}\right)_{M},\left(L_{3}\right)_{M}\right)
$$

and refer to it as the Leray invariant of $L_{1}, L_{2}, L_{3}$.

Theorem 2.11. Let $L_{i}, L_{i}^{\prime}, i=1,2,3$, be two triplets of Lagrangian subspaces of $X$. Then there exists a $\sigma \in \operatorname{Sp}(X)$, such that

$$
L_{i} \cdot \sigma=L_{i}^{\prime}, \quad \text { for } i=1,2,3
$$

if and only if the following relations are satisfied:

(1) $\operatorname{dim}\left(L_{1} \cap L_{2} \cap L_{3}\right)=\operatorname{dim}\left(L_{1}^{\prime} \cap L_{2}^{\prime} \cap L_{3}^{\prime}\right)$.

(2) $\operatorname{dim}\left(L_{i} \cap L_{j}\right)=\operatorname{dim}\left(L_{i}^{\prime} \cap L_{j}^{\prime}\right)$, for all $i, j$.

(3) $q\left(L_{1}, L_{2}, L_{3}\right)=q\left(L_{1}^{\prime}, L_{2}^{\prime}, L_{3}^{\prime}\right)$.

Proof. The necessity of the conditions being clear we consider sufficiency. Let $X=\sum X_{j}=\sum X_{j}^{\prime}$ be the decompositions of $X$ associated by Lemma 6 to the triples $L_{i}$ and $L_{i}^{\prime}$ respectively. Then it is easy to see that

$$
\begin{aligned}
& \operatorname{dim} X_{0}=2 \operatorname{dim}\left(L_{1} \cap L_{2} \cap L_{3}\right), \\
& \operatorname{dim} X_{1}=2 \operatorname{dim}\left(L_{2} \cap L_{3}\right)-\operatorname{dim} X_{0}, \\
& \operatorname{dim} X_{2}=2 \operatorname{dim}\left(L_{3} \cap L_{1}\right)-\operatorname{dim} X_{0}, \\
& \operatorname{dim} X_{3}=2 \operatorname{dim}\left(L_{1} \cap L_{2}\right)-\operatorname{dim} X_{0} .
\end{aligned}
$$

From this it is clear that $\operatorname{dim} X_{j}=\operatorname{dim} X_{j}^{\prime}$ for $0 \leq j \leq 4$. It is easy to see from the defining properties of the decomposition in Lemma 6, that there exists a symplectomorphism $\sigma_{j}: X_{j} \rightarrow X_{j}^{\prime}, j=0,1,2,3$, such that

$$
\left(L_{i} \cap X_{j}\right) \sigma_{j}=\left(L_{i}^{\prime} \cap X_{j}^{\prime}\right), \quad i=1,2,3,
$$

for each $j=0,1,2,3$. From Lemmas 4 and 7, it follows that there exists a symplectomorphism $\sigma_{4}: X_{4} \rightarrow X_{4}^{\prime}$ such that

$$
\left(L_{i} \cap X_{4}\right) \sigma_{4}=L_{i}^{\prime} \cap X_{4}^{\prime}, \quad i=1,2,3 .
$$

Let the map $\sigma: X \rightarrow X$, be defined by $\sigma \mid X_{j}=\sigma_{j}$. Then $\sigma \in \operatorname{Sp}(X)$ and $L_{i} \sigma=L_{i}^{\prime}$ for $i=1,2,3$. This completes the proof.

From the above theorem the following corollary is immediate. 
Corollary 2.12. Let $L_{1}, L_{2}, L_{3} \in \Lambda(X)$ and let $X=\sum X_{j}=$ $\sum X_{j}^{\prime}$ be two decompositions with the properties stated in Lemma 6, for the triple $L_{i}$. Then there exists $\sigma \in P_{L_{1}} \cap P_{L_{2}} \cap P_{L_{3}}$ such that $X_{j} \cdot \sigma=X_{j}^{\prime}$ for all $j$.

2.5. The group $W$. We fix two transversal Lagrangian subspaces $V, V^{*}$ of $X$ and bases $e_{1}, \ldots, e_{n}$ of $V$ and $e_{1}^{*}, e_{2}^{*}, \ldots, e_{n}^{*}$ of $V^{*}$ such that $\left\langle e_{i}, e_{j}^{*}\right\rangle=\delta_{i j}$. Thus $e_{1}, \ldots, e_{n}^{*}$ is a symplectic basis of $X$. Let

$$
W=\left\{\sigma \in \operatorname{Sp}(X) \mid\left\{e_{i}, e_{i}^{*}\right\} \sigma \subseteq\left\{ \pm e_{i}, \pm e_{i}^{*}\right\} \text { for all } i\right\} .
$$

Then clearly $W$ is a finite subgroup. Define the elements $\tau, \tau_{S}$, $a_{S} \in \operatorname{Sp}(X)$ as follows:

$$
e_{i} \cdot \tau=-e_{i}^{*}, \quad e_{i}^{*} \tau=e_{i} \text { for all } i .
$$

For a subset $S \subset\{1,2, \ldots, n\}$, let

$$
\begin{aligned}
& e_{i} \cdot \tau_{S}=\left\{\begin{array}{ll}
-e_{i}^{*}, & i \in S, \\
e_{i}, & i \notin S ;
\end{array} \quad e_{i}^{*} \cdot \tau_{S}= \begin{cases}e_{i}, & i \in S, \\
e_{i}^{*}, & i \notin S ;\end{cases} \right. \\
& e_{i} \cdot a_{S}=\left\{\begin{array}{ll}
-e_{i}, & i \in S, \\
e_{i}, & i \notin S ;
\end{array} \quad e_{i}^{*} \cdot a_{S}= \begin{cases}-e_{i}^{*}, & i \in S, \\
e_{i}^{*}, & i \notin S .\end{cases} \right.
\end{aligned}
$$

LEMMA 2.13. Any element $w$ of $W$ can be written uniquely in the form

$$
w=a_{S_{1}} \tau_{S_{2}}
$$

for some subsets $S_{1}, S_{2}$ of $\{1,2, \ldots, n\}$. Moreover the following relations hold: $\tau_{S}^{2}=a_{S}$ and $\tau_{S_{1} \cup S_{2}}=\tau_{S_{1}} \cdot \tau_{S_{2}}$ if $S_{1}, S_{2}$ are disjoint. In particular $W$ is a commutative group of order $2^{2 n}$. Every element of $W$ is of order at most 4 and the set of elements of order 2 is precisely the set $\left\{a_{s}\right\}$.

Proof. Let $\sigma \in W$. Then it is clear that $\sigma$ is of the form

$$
e_{i} \cdot \sigma=\left\{\begin{array}{ll}
-\varepsilon_{i} e_{i}^{*}, & i \in S, \\
\varepsilon_{i} e_{i}, & i \notin S ;
\end{array} \quad e_{i}^{*} \cdot \sigma= \begin{cases}\varepsilon_{i}^{\prime} e_{i}, & i \in B, \\
\varepsilon_{i}^{\prime}, e_{i}^{*}, & i \notin B .\end{cases}\right.
$$

The condition that $\sigma$ is symplectic now gives that $S=B$ and $\varepsilon_{i}=\varepsilon_{i}^{\prime}$ for all $i$. It is then clear that $\sigma=a_{S_{1}} \tau_{S_{2}}$ where $S_{2}=S$ and $S_{1}=\{i$ : $\left.\varepsilon_{i}=-1\right\}$. The rest of the statements follow easily from this.

REMARK. If $W_{S}$ is the subgroup of $\operatorname{Sp}\left(X_{S}\right)$ corresponding to the data, $X_{S}=V_{S}+V_{S}^{*}$ and the symplectic basis $\left\{e_{i}, e_{i}^{*}, i \in S\right\}$ then $W_{S}$ is just the group obtained by restricting $W$ to the subspace $X_{S}$. 
2.6. The P-double cosets. From now on we write

$$
P=P_{V^{*}}, \quad N=N_{V^{*}} .
$$

Thus in the decomposition $X=V+V^{*}$, the elements of $P$ and $N$ have the matrix representations of the form

$$
\left[\begin{array}{ll}
\alpha & \beta \\
0 & \delta
\end{array}\right], \quad\left[\begin{array}{ll}
I & \rho \\
0 & I
\end{array}\right]
$$

respectively. The following lemma is known. It describes the $P$ double cosets in $\operatorname{Sp}(X)$.

LEMMA 2.14. Let

$$
\Omega_{j}=\left\{\sigma \in \operatorname{Sp}(X) \mid \operatorname{dim} V^{*} \cap V^{*} \sigma=n-j\right\}, \quad 0 \leq j \leq n .
$$

Then $\operatorname{Sp}(X)=\bigcup \Omega_{j}$. Each $\Omega_{j}$ is a single double coset of $P$. Moreover

$$
\Omega_{j}=\left\{\sigma=\left[\begin{array}{ll}
\alpha & \beta \\
\gamma & \delta
\end{array}\right] \in \operatorname{Sp}(X) \mid \operatorname{dim} \operatorname{ker} \gamma=n-j\right\}
$$

Proof. Since $P$ is the stabilizer of $V^{*}$, it is clear that $P \Omega_{j} P=\Omega_{j}$. Now suppose $\sigma_{1}, \sigma_{2} \in \Omega_{j}$. Then from Lemma 2.3, it follows that $V^{*} \sigma_{1} p=V^{*} \sigma_{2}$ for some $p \in P$. Thus $\sigma_{1} p \sigma_{2}^{-1}$ stabilizes $V^{*}$ and also in $P$. Thus $\sigma_{1} \in P \sigma_{2} P$, i.e. $\Omega_{j}$ is a single $P$-double coset. For the last part note that $\operatorname{dim} V^{*} \cap V^{*} \sigma=\operatorname{dim} V^{*} \cap V^{*} \sigma^{-1}$ and

$$
V^{*} \cap V^{*} \sigma^{-1}=\operatorname{ker} \gamma, \quad \text { if } \sigma=\left[\begin{array}{ll}
\alpha & \beta \\
\gamma & \delta
\end{array}\right] \text {. }
$$

This completes the proof.

LEMMA 2.15. (i) $V^{*} \cap V^{*} \tau_{S}=V^{*} \cap V^{*} \sigma_{S}^{-1}=V_{S}^{*}$, where $S^{\prime}$ is the complement of $S$.

(ii) $\tau_{S} \in \Omega_{j}$, where $j$ is the number of elements in $S$. In particular $\operatorname{Sp}(X)=P W P$.

(iii) If $w_{j}=a_{A_{j}} \tau_{B_{j}}, j=1,2$, then $w_{1}, w_{2}$ belong to the same $P$-double coset if and only if the number of elements in the sets $B_{1}, B_{2}$ are the same.

The proof is straightforward and is omitted.

The following theorem plays a central role in the description of the multiplier of the Segal-Shale-Weil representation. 
Theorem 2.16. Let $\sigma_{1}, \sigma_{2} \in \operatorname{Sp}(X)$. Then there exist suitable $p_{1}, p_{2}, p \in P$ such that

$$
\begin{aligned}
& \sigma_{1}=p_{1} \kappa_{1} p^{-1}, \quad \sigma_{2}=p \kappa_{2} p_{2}, \\
& \kappa_{1}=\operatorname{diag}\left(\tau_{S_{1}}, \tau_{S} u_{\rho}\right)=\tau_{S_{1} \cup S} \cdot u_{\rho}, \\
& \kappa_{2}=\operatorname{diag}\left(\tau_{S_{2}}, \tau_{S}\right)=\tau_{S_{2} \cup S},
\end{aligned}
$$

where $S_{1}$ and $S_{2}$ are contained in the complement $S^{\prime}$ of $S$ and the $\operatorname{diag}(\cdot, \cdot)$ is relative to the decomposition $X=X_{S^{\prime}}+X_{S}$. Here $\rho$ is a nondegenerate symmetric map of $V_{S}$ onto $V_{S}^{*}$, whose isometry class is that given by the Leray invariant $-q\left(V^{*}, V^{*} \sigma_{1}, V^{*} \sigma_{2}^{-1}\right)$.

Proof. Let $L_{1}=V^{*}, L_{2}=V^{*} \sigma_{2}^{-1}, L_{3}=V^{*} \sigma_{1}$. Let $j_{1}=\operatorname{dim} V^{*} \cap$ $V^{*} \sigma_{1}, j_{2}=\operatorname{dim} V^{*} \cap V^{*} \sigma_{2}$ and $j=\operatorname{dim} V^{*} \cap V^{*} \sigma_{1} \sigma_{2}, j_{0}=\operatorname{dim} V^{*} \cap$ $V^{*} \sigma_{1} \cap V^{*} \sigma_{2}^{-1}$. Choose a partition $B_{0}, \ldots, B_{4}$ of $\{1,3, \ldots, n\}$ with $j_{0}, j-j_{0}, j_{1}-j_{0}, j_{2}-j_{0}, n+2 j_{0}-\left(j_{1}+j_{2}+j\right)$ elements respectively. Let $X_{i}=X_{B_{i}}$ and $V_{i}=V_{B_{i}}, V_{i}^{*}=V_{B_{i}}^{*}$. Let $\rho$ be a nondegenerate symmetric map of $V_{4}$ onto $V_{4}^{*}$. In the decomposition $X=\sum X_{i}$, let $\kappa_{1}, \kappa_{2}$ be defined as follows:

$$
\kappa_{1}=\operatorname{diag}\left(I, \tau, I, \tau, \tau u_{\rho}\right), \quad \kappa_{2}=\operatorname{diag}(I, \tau, \tau, I, \tau)
$$

where the diagonal components belong to $\operatorname{Sp}\left(X_{i}\right)$ respectively and $\tau$ as a diagonal component is to be understood as $\tau$ restricted to the appropriate $\operatorname{Sp}\left(X_{i}\right)$. Let $L_{1}^{\prime}=V^{*}, L_{2}^{\prime}=V^{*} \kappa_{2}^{-1}, L_{3}^{\prime}=V^{*} \kappa_{1}$. Then it is clear that $\operatorname{dim} L_{1} \cap L_{2} \cap L_{3}=\operatorname{dim} L_{1}^{\prime} \cap L_{2}^{\prime} \cap L_{3}^{\prime}$ and $\operatorname{dim} L_{i} \cap L_{j}=$ $\operatorname{dim} L_{i}^{\prime} \cap L_{j}^{\prime}$, for all $i, j$. If we now choose the map $\rho$ such that the isometry class of $\langle x, x \cdot \rho\rangle$ on $V_{4}$ is equal to $q\left(L_{1}, L_{2}, L_{3}\right)$, then by Theorem 1 , there exists $p \in P$, such that

$$
V^{*} \sigma_{2}^{-1} p=V^{*} \kappa_{2}^{-1}, \quad V^{*} \sigma_{1} p=V^{*} \kappa_{1} .
$$

Thus

$$
\sigma_{2}^{-1} p \kappa_{2} \in P, \quad \sigma_{1} p \kappa_{1}^{-1} \in P .
$$

If $S_{1}=B_{1} \cup B_{3}, S_{2}=B_{1} \cup B_{2}$ and $S=B_{4}$, then $\kappa_{1}, \kappa_{2}$ have the form stated in the theorem and this completes the proof.

REMARK. From the above theorem it is easy to check that

$$
\Omega_{j_{1}} \boldsymbol{\Omega}_{j_{2}}=\bigcup_{j \leq j_{1}+j_{2}} \boldsymbol{\Omega}_{j}
$$

In particular $\Omega_{n}^{2}=\operatorname{Sp}(X)$. Also note $\Omega_{j}^{-1}=\Omega_{j}$. 
2.7. An example. Let $\operatorname{dim} X=2$ and suppose $e_{1}, e_{2}$ is a symplectic basis, so that $\left\langle a_{1} e_{1}+a_{2} e_{2}, b_{1} e_{1}+b_{2} e_{2}\right\rangle=a_{1} b_{2}-a_{2} b_{1}$. Let $V=k \cdot e_{1}, V^{*}=k \cdot e_{2}$. Now Lagrangian subspaces are just lines in $X$ and we shall get an expression for the Leray invariant of three lines $L_{1}, L_{2}, L_{3}$. Since the Leray invariant is trivial when two of them coincide, we assume that they are all distinct. Let $x_{j} \in L_{j}$ be non-zero points. Then there exist scalars $a, b$ such that $x_{3}=a x_{2}+a b x_{1}$. If $\rho: L_{2} \rightarrow L_{1}$, defined as $\rho: t x_{2} \rightarrow t b x_{1}$, then $L_{3}=\left\{x+x \rho: x \in L_{2}\right\}$. Thus the associated quadratic form $t \rightarrow\left\langle t x_{2}, t x_{2} \cdot \rho\right\rangle=t^{2} b\left\langle x_{2}, x_{1}\right\rangle$. One gets from this that $q\left(L_{1}, L_{2}, L_{3}\right)$ is the isometry class of the form $\left\langle\rho t^{2}\right\rangle$ where

$$
\rho=-\left\langle x_{1}, x_{2}\right\rangle\left\langle x_{2}, x_{3}\right\rangle\left\langle x_{3}, x_{1}\right\rangle\left(k^{x}\right)^{2} .
$$

Now suppose $\sigma_{1}, \sigma_{2} \in \operatorname{Sp}(X, k)$ and

$$
\sigma_{j}=\left[\begin{array}{ll}
a_{j} & b_{j} \\
c_{j} & d_{j}
\end{array}\right]
$$

for $j=1,2$. Then the above calculation leads to the following result:

$$
q\left(V^{*}, V^{*} \sigma_{2}^{-1}, V^{*} \sigma_{1}\right)=\left\{\begin{array}{l}
\text { trivial if } \sigma_{1}, \sigma_{2} \text { or } \sigma_{1} \sigma_{2} \in P, \\
c_{1} c_{2}\left(c_{1} a_{2}+d_{1} c_{2}\right) \cdot\left(k^{x}\right)^{2}, \quad \text { otherwise }
\end{array}\right.
$$

here one has identified the nontrivial isometry classes with $k^{x} /\left(k^{x}\right)^{2}$.

\section{The standard form for the Segal-Shale-Weil representation.}

3.1. Throughout the rest of the paper we assume that (1) $F$ is a selfdual locally compact field, i.e. $F$ is either a finite field or a nondiscrete locally compact field, (2) char $F \neq 2$. It should be noted that in $\S 2$, the field $F$ was allowed to have characteristic 2 . It is possible to generalize the results of this section also to the case of char $k=2$, although the representation obtained is not of the symplectic group, but of the pseudosymplectic group. For simplicity of presentation we shall assume however that $\operatorname{char} F \neq 2$.

We fix the following notation: $X$ is a symplectic vector space over $F, V, V^{*}$ two transversal Lagrangian subspaces of $X, e_{1}, \ldots, e_{n}^{*}$ is a symplectic basis of $X$ such that $e_{1}, \ldots, e_{n}\left(e_{1}^{*}, \ldots, e_{n}^{*}\right)$ is a basis of $V\left(V^{*}\right) ; \chi$ is a nontrivial continuous unitary character of the additive group of $F ; d_{F}^{+} x$ denotes the Haar measure of the additive group of $F$ which is self-dual relative to the pairing $a, b \rightarrow \chi(a b)$ of $F$ with itself. Let $V_{S}, V_{S}^{*}$ denote the subspaces spanned by $\left\{e_{j}\right.$, $j \in S\}$ and $\left\{e_{j}^{*}, j \in S\right\}$ respectively. Here $S$ is an arbitrary subset of $\{1,2, \ldots, n\}$. Then $V_{S}, V_{S}^{*}$ are locally compact groups which 
may be considered as character groups of each other, via the pairing $v, v^{*} \rightarrow \chi\left(\left\langle v, v^{*}\right\rangle\right)$. Let $d_{V_{S}}, d_{V_{S}^{*}}$ denote the Haar measure on $V_{S}$, $V_{S}^{*}$ respectively defined as the product Haar measures. For instance

$$
\int_{V_{S}} f(v) d_{V_{s}} v=\int f\left(\sum_{j \in S} x_{j} e_{j}\right) \prod_{j \in S} d_{F}^{+} x_{j}
$$

Then the Haar measures $d_{V_{S}}$ and $d_{V_{S}^{*}}$ are dual to each other relative to the pairing $\chi$ introduced above. Let $\mathscr{S}(V), \mathscr{S}\left(V_{S}\right)$ etc. denote the space of Bruhat-Schwartz functions on $V, V_{S}$ etc. Then it is known and easy to check that $\mathscr{S}\left(V_{S_{1}}\right) \otimes \mathscr{S}\left(V_{S_{2}}\right)=\mathscr{S}\left(V_{S_{1} \cup S_{2}}\right)$ if $S_{1} \cap S_{2}=\varnothing$ (see for instance Bruhat [2]). The Fourier transforms $\mathscr{F}_{S}: \mathscr{S}\left(V_{S}\right) \rightarrow$ $\mathscr{S}\left(V_{S}^{*}\right), \mathscr{F}_{S}^{*}: \mathscr{S}\left(V_{S}^{*}\right) \rightarrow \mathscr{S}\left(V_{S}\right)$ are defined as:

$$
\begin{gathered}
\mathscr{F}_{S} \varphi: x^{*} \mapsto \int_{V_{S}} \chi\left(\left\langle x, x^{*}\right\rangle\right) \varphi(x) d_{V_{S}} x, \\
\mathscr{F}^{*} \psi: x \rightarrow \int_{V_{S}^{*}} \chi\left(\left\langle x, x^{*}\right\rangle\right) \psi\left(x^{*}\right) d_{V_{S}^{*}} x^{*}
\end{gathered}
$$

for all $\varphi \in \mathscr{S}\left(V_{S}\right), \psi \in \mathscr{S}\left(V_{S}^{*}\right)$. The Haar measures $d_{V_{S}}, d_{V_{S}^{*}}$ being dual to each other is equivalent to saying that $\mathscr{F}_{S}^{*} \mathscr{F}_{S} \varphi=\varphi^{\circ}$, $\mathscr{F}_{S} \mathscr{F}_{S}{ }^{*} \psi=\psi^{\circ}$ where $\varphi^{\circ}(x)=\varphi(-x), \psi^{\circ}$ being defined similarly.

3.2. We next recall the definition of the projective representation of symplectic groups known as the Segal-Shale-Weil representation. ${ }^{1}$ (The basic references are Weil [16], Shale [15], Segal [13]; see Mackey [10], for a historical survey and also Gelbart [4], for further references.) Let $U=U_{V, V^{*}}$ denote the projective unitary representation of $X$ in $L^{2}(V)$ defined as follows:

$$
U\left(v+v^{*}\right) \varphi: x \mapsto \chi\left(\left\langle x, v^{*}\right\rangle\right) \varphi(x+v) .
$$

If $w_{j}=v_{j}+v_{j}^{*} \in X, j=1,2$, then

$$
U\left(w_{1}\right) U\left(w_{2}\right)=\chi\left(\left\langle v_{1}, v_{2}^{*}\right\rangle\right) U\left(w_{1}+w_{2}\right) .
$$

Let $H=H\left(V, V^{*}, \chi\right)$ denote the Heisenberg group defined as $H=$ $X \times T$ with group law

$$
\left(w_{1}, t_{1}\right) \circ\left(w_{2}, t_{2}\right)=\left(w_{1}+w_{2}, t_{1} t_{2} \chi\left(\left\langle v_{1}, v_{2}^{*}\right\rangle\right)\right) \text {. }
$$

If

$$
\widetilde{U}(w, t)=t U(w)
$$

${ }^{1}$ It is also referred to in the literature as the Weil representation, or Shale-Weil representation, the oscillator representation or the metaplectic representation. We follow Weil's paper rather closely. 
then $U$ is an irreducible, unitary representation of $H$ with central character $\chi$. According to Stone-von Neumann there is only one such unitary representation of $H$ up to unitary equivalence. Now let $\operatorname{Ps}(H)$ denote the pseudosymplectic group of $H$, i.e. the set of all continuous automorphisms of $H$ which leave the center element-wise fixed. Let $\widetilde{U}^{s}:(w, t) \rightarrow \widetilde{U}((w, t) \cdot s), s \in \operatorname{Ps}(H)$. Then it follows from the Stone-von Neumann Theorem that there exists a unitary operator $\xi_{s}$, such that

$$
\xi_{s}^{-1} \circ \tilde{U} \circ \xi_{s}=\widetilde{U}^{s} .
$$

The operators $\xi_{s}$ satisfying (3.5) are unique up to a scalar multiple. Thus any choice $s \rightarrow \xi_{s}$ of such operators gives rise to a Weil (projective) representation of the group $\operatorname{Ps}(H)$. Let $s \in \operatorname{Ps}(H)$, and suppose $(w, 1) s=(w \cdot \sigma, f(w))$; then $(\sigma, f)$ parametrize $\operatorname{Ps}(H)$ and are characterized by the properties

(i) $\sigma$ is a continuous automorphism of the additive group of $X$, which leaves the form $\langle\cdot, \cdot\rangle$ invariant;

(ii) $f: X \rightarrow T$ is a continuous map, such that

$$
f\left(w_{1}+w_{2}\right)\left\{f\left(w_{1}\right)\right\}^{-1}\left\{f\left(w_{2}\right)\right\}^{-1}=\chi\left(\left\langle v_{1 \sigma}, v_{2 \sigma}^{*}\right\rangle\right)\left\{\chi\left(\left\langle v_{1}, v_{2}^{*}\right\rangle\right)\right\}^{-1}
$$

for all $w_{j}=v_{j}+v_{j}^{*} \in X$, where $w_{j} \cdot \sigma=v_{j \sigma}+v_{j \sigma}^{*}, j=1,2$.

The subgroup

$$
\operatorname{Ps}(H, F)=\{(\sigma, f) \in \operatorname{Ps}(H) \mid \sigma \text { is } F \text {-linear }\}
$$

is called the linear pseudosymplectic group. When $F=\mathbb{R}$ or $Q_{p}$ it coincides with $\operatorname{Ps}(H)$ and is smaller for all other $F$. If $X^{*}$ denotes the character group of $X$, then the subgroup $\{(1, f) \in \operatorname{Ps}(H)\}$ may be identified with $X^{*}$ and the sequence

$$
0 \rightarrow X^{*} \rightarrow \operatorname{Ps}(H, F) \rightarrow \operatorname{Sp}(X) \rightarrow 0
$$

is exact (see [16], p. 150). Moreover since we have assumed that char $F \neq 2$, the exact sequence splits. In fact when char $F \neq 2$, the following lemma gives a splitting homomorphism of $\operatorname{Sp}(X)$ into $\operatorname{Ps}(H, F)$ (see Weil [16], p. 150).

LeMma 3.1. Assume char $F \neq 2$. Let $\sigma \in \mathrm{Sp}(X)$ have the matrix representation

$$
\sigma=\left[\begin{array}{ll}
\alpha & \beta \\
\gamma & \delta
\end{array}\right]
$$


in the decomposition $X=V+V^{*}$. Let

$$
\begin{gathered}
q_{\sigma}\left(v+v^{*}\right)=\frac{1}{2}\langle v \alpha, v \beta\rangle+\frac{1}{2}\left\langle v^{*} \gamma, v^{*} \delta\right\rangle+\left\langle v^{*} \gamma, v \beta\right\rangle, \\
f_{\sigma}\left(v+v^{*}\right)=\chi\left(q_{\sigma}\left(v+v^{*}\right)\right) .
\end{gathered}
$$

Then $\sigma \rightarrow\left(\sigma, f_{\sigma}\right)$ is a homomorphism of $\operatorname{Sp}(X)$ into $\operatorname{Ps}(H, F)$. In particular a mapping $\sigma \rightarrow \xi_{\sigma}$ of $\mathrm{Sp}(X)$ into unitary operators in $L^{2}(V)$ is a Weil (projective) representation if

$$
\xi_{\sigma}^{-1} U\left(v+v^{*}\right) \xi_{\sigma}=f_{\sigma}\left(v+v^{*}\right) U\left(\left(v+v^{*}\right) \cdot \sigma\right)
$$

for all $v+v^{*} \in X$.

3.3. Our next object is to present an explicit integral formula for the operators $\xi_{\sigma}$ satisfying (3.6). This is essentially known and in fact Weil himself has given this when the matrix entry $\gamma$ of $\sigma$ in (3.6) is either an isomorphism or 0. However for our purposes it is important to do it for all $\sigma$. After this work was done, the author came across the paper of Lions [9], where a related formula for intertwining operators of unitary representations of a real nilpotent group is given. We shall present a proof based on Bruhat decomposition of $\operatorname{Sp}(X)$ (see Lemma 2.14) and it would appear that this was the way Weil derived his general formula also. For the meaning of the symbols $P=P_{V^{*}}, N=N_{V^{*}}, \tau, \tau_{S}$ etc. see $\S 2.5$.

Lemma 3.2. (1) Let $p \in P$. Then the operator $\xi_{\sigma}$ satisfying (3.6) is a scalar multiple of $r(p)$, where

$$
r(p) \varphi: x \rightarrow|\alpha|^{1 / 2} f_{p}(x) \varphi(x \alpha) .
$$

(2) Let $S$ be an arbitrary subset of $\{1,2, \ldots, n\}$; the operator $\xi_{\sigma}$, for $\sigma=\tau_{S}$, is a scalar multiple of $r\left(\tau_{S}\right)$, where $r\left(\tau_{S}\right)$ is the partial Fourier transform

$$
r\left(\tau_{S}\right) \varphi: x \rightarrow \int_{V_{S}} \chi\left(\left\langle y, x_{S} \tau\right\rangle\right) \varphi\left(x_{S^{\prime}}+y\right) d_{V_{S}} y
$$

where $S^{\prime}$ is the complement of $S$ in $\{1,2, \ldots, n\}$ and $x=x_{S}+x_{S^{\prime}}$ in the decomposition $V=V_{S}+V_{S^{\prime}}$.

(3) For a general $\sigma \in \operatorname{Sp}(X)$, let $M_{\sigma}=V^{*} / \operatorname{ker} \gamma$, and $\mu_{\sigma}$ denote a Haar measure on $M_{\sigma}$. Let

$$
T_{\sigma} \varphi: x \rightarrow \int_{M_{\sigma}} f_{\sigma}\left(x+x^{*}\right) \varphi\left(x \alpha+x^{*} \gamma\right) \mu_{\sigma}\left(d \bar{x}^{*}\right)
$$


where $\bar{x}^{*}$ is the coset $x^{*}+\operatorname{ker} \gamma$. Then for $\varphi \in \mathscr{S}(V)$, the integral is absolutely convergent and $T_{\sigma} \varphi \in \mathscr{S}(V)$. Moreover $T_{\sigma}$ satisfies the identity

$$
T_{\sigma} U\left(v+v^{*}\right) \varphi=f_{\sigma}\left(v+v^{*}\right) U\left(\left(v+v^{*}\right) \sigma\right) T_{\sigma} \varphi
$$

for all $\varphi \in \mathscr{S}(V)$. In particular for a suitable choice of Haar measures $\mu_{\sigma}$, the map $\sigma \rightarrow T_{\sigma}$ is a Weil (projective) representation.

Proof. The statement (i) and (2) are straightforward to verify. In fact (i) and (ii) when $\tau_{S}=\tau$ is already present in [16]. For a general subset $S$, note that $X=X_{S}+X_{S^{\prime}}$ and $\tau_{S}=\operatorname{diag}(\tau, I)$ and the formula follows from that for $\tau$. From Bruhat decomposition Lemma 2.14 it follows that operators satisfying the identity (3.6) will leave the Schwartz space invariant.

To prove (3), note the integrand is a well-defined function of the coset $x^{*}+\operatorname{ker} \gamma$. For this one has only to check $f_{\sigma}\left(x+x^{*}+z^{*}\right)=$ $f_{\sigma}\left(x+x^{*}\right)$ if $z^{*} \in \operatorname{ker} \gamma$. In fact this is clear from the formula for $f_{\sigma}$ given in Lemma 3.1. Since $\gamma$ is $F$-linear, the image of $\gamma$ is a linear subspace $E_{\sigma}$ of $V$ and is thus isomorphic to $M_{\sigma}$. From this we have the estimate

$$
\left|T_{\sigma} \varphi \cdot(x)\right| \leq \int_{E_{\sigma}}|\varphi(x+y)| d_{E} y
$$

where $d_{E_{\sigma}} y$ is a Haar measure on $E_{\sigma}$. Thus $T_{\sigma} \varphi$ is a continuous function of $x$. The verification of the identity (3.7) is straightforward and so is omitted. If therefore $A_{\sigma}=T_{\sigma} \xi_{\sigma}^{-1}$, where $\xi_{\sigma}$ is a unitary operator leaving the space $\mathscr{S}(V)$ invariant and satisfying (3.7), then $A_{\sigma}: \mathscr{S}(V) \rightarrow C(V)$ is a linear operator which commutes with $U$. From standard arguments it now follows that $A_{\sigma}$ is a scalar. This completes the proof.

LEMMA 3.3. With the notation of the above lemma, we have

(1) If $p \in P$, then $M_{p}=\{0\}$ and $T_{p}=r(p)$, provided $\mu_{p}\{0\}=$ $\left|\operatorname{deg}\left(p \mid V^{*}\right)\right|^{-1 / 2}$.

(2) $M_{\sigma p}=M_{\sigma}$, and $T_{\sigma p}=T_{\sigma} r(p)$ if

$$
\mu_{\sigma p}^{\prime}=\left|\operatorname{deg}\left(p \mid V^{*}\right)\right|^{-1 / 2} \mu_{\sigma} .
$$

(3) The map $x^{*} \rightarrow x^{*} p$ of $V^{*}$ onto $V^{*}$ factors down to a map $\bar{x}^{*} \rightarrow \bar{x}^{*} \bar{p}$ of $M_{p \sigma}$ onto $M_{\sigma}$. Then $T_{p \sigma}=r(p) T_{\sigma}$ if and only if ${ }^{2}$

$$
\mu_{p \sigma}=\left|\operatorname{deg}\left(p \mid V^{*}\right)\right|^{-1 / 2} \bar{p} \cdot \mu_{\sigma} .
$$

\footnotetext{
${ }^{2}$ Here for a measure $\nu$ on $M_{p \sigma},(\bar{p})^{-1} \cdot \nu$ is the measure on $M_{\sigma}$, defined as the linear form $\varphi \rightarrow \nu(\varphi \circ \bar{p})$ for functions $\varphi$ on $M_{\sigma}$.
} 
Proof. (i) This follows from $r(p) \varphi \cdot(0)=\left|\operatorname{deg}\left(p \mid V^{*}\right)\right|^{-1 / 2} \varphi(0)$ while $T_{p} \varphi \cdot(0)=\mu_{p}\{0\} \varphi(0)$.

(ii) Note $\operatorname{ker} \gamma(\sigma)=V^{*} \cap V^{*} \sigma^{-1}$. Thus $\operatorname{ker} \gamma(\sigma p)=\operatorname{ker} \gamma(\sigma)$. Thus $M_{\sigma p}=M_{\sigma}$. On the one hand

$$
T_{\sigma} r(p) \varphi \cdot(0)=\left|\operatorname{deg}\left(p \mid V^{*}\right)\right|^{-1 / 2} \int_{M_{\sigma}} f_{\sigma}\left(x^{*}\right) f_{p}\left(x^{*} \gamma\right) \varphi\left(x^{*} \gamma p_{11}\right) \mu_{\sigma}\left(d \bar{x}^{*}\right)
$$

where

is the matrix of $p$. Next

$$
p \rightarrow\left[\begin{array}{cc}
p_{11} & p_{12} \\
0 & p_{22}
\end{array}\right]
$$

$$
T_{\sigma p} \varphi \cdot(0)=\int_{M_{\sigma p}} f_{\sigma p}\left(x^{*}\right) \varphi\left(x^{*} \gamma p_{11}\right) \mu_{\sigma p}\left(d \bar{x}^{*}\right) .
$$

Now

$$
f_{\sigma p}\left(x^{*}\right)=f_{\sigma}\left(x^{*}\right) f_{p}\left(x^{*} \gamma\right)
$$

since $\sigma \rightarrow\left(\sigma, f_{\sigma}\right)$ is a homomorphism. From this the result follows.

(iii) One checks directly from the matrix representation (3.6) of $\sigma$, that $\gamma(p \sigma)=p \gamma(\sigma)$. Thus the map $x^{*} \rightarrow x^{*} \cdot p$ takes $\operatorname{ker} \gamma(p \sigma)$ to $\operatorname{ker} \gamma(\sigma)$. Thus the map

$$
\bar{p}: x^{*}+\operatorname{ker} \gamma(p \sigma) \rightarrow x^{*} \cdot p+\operatorname{ker} \gamma(\sigma)
$$

maps $M_{\sigma p}$ onto $M_{\sigma}$. Now

$$
\begin{aligned}
r(p) T_{\sigma} \varphi \cdot(0) & =\left|\operatorname{deg}\left(p \mid V^{*}\right)\right|^{-1 / 2} T_{\sigma} \varphi \cdot(0) \\
& =\left|\operatorname{deg}\left(p \mid V^{*}\right)\right|^{-1 / 2} \int_{M_{\sigma}} f_{\sigma}\left(x^{*}\right) \varphi\left(x^{*} \gamma\right) \mu_{\sigma}\left(d \bar{x}^{*}\right) .
\end{aligned}
$$

On the other hand

$$
T_{p \sigma} \varphi \cdot(0)=\int_{M_{p \sigma}} f_{p \sigma}\left(x^{*}\right) \varphi\left(x^{*} p \gamma\right) \mu_{\sigma p}\left(d \bar{x}^{*}\right) .
$$

From the homomorphism property of $\sigma \rightarrow\left(\sigma, f_{\sigma}\right)$ we have

$$
f_{p \sigma}\left(x^{*}\right)=f_{p}\left(x^{*}\right) f_{\sigma}\left(x^{*} p\right)=f_{\sigma}\left(x^{*} p\right) \text {. }
$$

Thus

$$
T_{p \sigma} \varphi \cdot(0)=\int_{M_{p \sigma}} f_{\sigma}\left(x^{*} p\right) \varphi\left(x^{*} p \gamma\right) \mu_{p \sigma}\left(d \bar{x}^{*}\right) .
$$

Comparing the formulas for $T_{p \sigma} \varphi \cdot(0)$ and $T_{p} T_{\sigma} \varphi \cdot(0)$ one gets

$$
\left|\operatorname{deg}\left(p \mid V^{*}\right)\right|^{-1 / 2} \int_{M_{\sigma}} \psi(\bar{x}) \mu_{\sigma}(d \bar{x})=\int_{M_{p \sigma}} \psi(\bar{y} \cdot \bar{p}) \mu_{p \sigma}(d \bar{y}) .
$$

This completes the proof.

The following lemma makes it possible to make consistent choices of $\mu_{\sigma}$. 
Lemma 3.4. Let $\sigma \in \operatorname{Sp}(X)$ be arbitrary. Suppose $p_{1}, p_{2} \in P$ are such that $p_{1} \sigma p_{2}=\sigma$. Then $\bar{p}_{1}$ maps $M_{\sigma}$ onto itself and

$$
\left\{\operatorname{det}\left(\bar{p}_{1} \mid M_{\sigma}\right)\right\}^{2}=\operatorname{det}\left(p_{1} p_{2} \mid V^{*}\right) \text {. }
$$

Proof. Our first step is the observation that if the lemma is true for $\sigma$ it is also true for $\sigma^{\prime} \in P \sigma P$. In fact suppose $\sigma^{\prime}=m_{1} \sigma m_{2}$ and suppose $p_{1}^{\prime} \sigma^{\prime} p_{2}^{\prime}=\sigma^{\prime}$. Then $p_{1} \sigma p_{2}=\sigma$ where $p_{1}=m_{1}^{-1} p_{1}^{\prime} m_{1}$, $p_{2}=m_{2} p_{2}^{\prime} m_{2}^{-1}$. On the one hand since the lemma is true for $\sigma$,

$$
\left\{\operatorname{det}\left(\bar{p}_{1} \mid M\right)\right\}^{2}=\operatorname{deg}\left(p_{1} p_{2} \mid V^{*}\right)=\operatorname{det}\left(p_{1}^{\prime} p_{2}^{\prime} \mid V^{*}\right) .
$$

On the other hand we have the commuting diagram

$$
\begin{gathered}
M_{\sigma^{\prime}} \stackrel{\bar{p}_{1}^{\prime}}{\longrightarrow} M_{\sigma^{\prime}} \\
m_{1} \mid \stackrel{m_{1}}{ } \\
M_{\sigma} \stackrel{\bar{p}_{1}}{\longrightarrow} M_{\sigma}
\end{gathered}
$$

where the map of $M_{\sigma^{\prime}}$ to $M_{\sigma}$ is the map $x^{*}+V^{*} \cap V^{*} \sigma^{\prime-1} \rightarrow x^{*} m_{1}+$ $V^{*} \cap V^{*} \sigma^{-1}$. From this it follows that

$$
\operatorname{det}\left(\bar{p}_{1} \mid M_{\sigma}\right)=\operatorname{det}\left(\bar{p}_{1}^{\prime} \mid M_{\sigma}\right)
$$

so that the lemma is valid for $\sigma^{\prime}$. To prove the lemma it is thus sufficient to prove it when $\sigma=\tau_{S}$ for some $S$. Now $V^{*} \tau_{S}=V_{S}+V_{S^{\prime}}^{*}$, $S^{\prime}$ being the complement of $S$. Note $V^{*} \tau_{S}=V^{*} \tau_{S}^{-1}$. Let $P_{o}=$ $P \cap P_{V^{*} \sigma}$. If $p_{1} \tau_{S} p_{2}=\tau_{S}$, then $p_{1}, p_{2} \in P_{0}$. Now the matrix for $\tau_{S}$ in the decomposition

$$
X=V_{S^{\prime}}+V_{S}+V_{S}^{*}+V_{S^{\prime}}^{*}
$$

is

$$
\tau_{S}=\left[\begin{array}{llll}
I & 0 & 0 & 0 \\
0 & 0 & \tau & 0 \\
0 & \tau & 0 & 0 \\
0 & 0 & 0 & I
\end{array}\right]
$$

Here we have simply written $\tau$, rather than $\left(\tau \mid V_{S}\right),\left(\tau \mid V_{S}^{*}\right)$ etc. If $p, p^{\prime} \in P_{0}$, then they leave the flag $X \supset V_{S}+V^{*} \supset V^{*} \supset V_{S^{\prime}}^{*}$ invariant, so that their matrices in the above decomposition are upper triangular. Thus if $p \cdot \tau_{S}=\tau_{S} \cdot p^{\prime}$, and suppose $p=\left(a_{i j}\right), p^{\prime}=\left(b_{i j}\right)$, then both the matrices are upper triangular. The equation $p \tau_{S}=\tau_{S} p^{\prime}$ gives

$$
a_{11}=b_{11}, \quad a_{22} \tau=\tau b_{33}, \quad a_{33} \tau=\tau b_{22}, \quad a_{44}=b_{44} .
$$


Now

$$
\operatorname{det}\left(p \mid V^{*}\right)\left\{\operatorname{det}\left(p^{\prime} \mid V^{*}\right)\right\}^{-1}=\operatorname{det} a_{33} \operatorname{det} a_{44} \operatorname{det} b_{11} \operatorname{det} b_{22} .
$$

From the symplectic property of $p, p^{\prime}$ we have $b_{11}\left({ }^{t} b_{44}\right)=\mathrm{id}$; thus $\operatorname{det} b_{44} \operatorname{det} b_{11}=1$, while $\operatorname{det} b_{22}=\operatorname{det} a_{33}$, since $\tau^{-1} a_{33} \tau=b_{22}$. Thus we have

$$
\operatorname{det}\left(p \mid V^{*}\right)\left\{\operatorname{det}\left(p^{\prime} \mid V^{*}\right)\right\}^{-1}=\left(\operatorname{det} a_{33}\right)^{2} .
$$

Now $V^{*} \cap V^{*} \tau_{S}=V_{S^{\prime}}^{*}$. From this it follows that

$$
\operatorname{det}\left(\bar{p} \mid V_{S}^{*}\right)=\left(\operatorname{det} a_{33}\right) \text {. }
$$

This completes the proof.

The following is the main theorem of this section.

THEOREM 3.5. There exists a unique choice of unitary operators $r(\sigma), \sigma \in \operatorname{Sp}(X)$ with the following properties:

(1) $r(\sigma)^{-1} U(w) r(\sigma)=f_{\sigma}(w) U(w \cdot \sigma)$ for all $w \in X$, or $\sigma \rightarrow r(\sigma)$ is a Weil (projective) representation

(2) $r(p) \varphi: x \rightarrow|\alpha|^{1 / 2} f_{p}(x) \varphi(x \alpha)$, when $p \in P$.

(3) $r\left(p_{1} \sigma p_{2}\right)=r\left(p_{1}\right) r(\sigma) r\left(p_{2}\right)$ for all $p_{1}, p_{2} \in P$ and $\sigma$ arbitrary.

(4) $r\left(\sigma_{1} \sigma_{2}\right)=r\left(\sigma_{1}\right) r\left(\sigma_{2}\right)$ whenever $\sigma_{1}, \sigma_{2} \in W$ or $r \mid W$ is a representation of the finite group $W$.

(5) $r(\sigma) \psi \cdot(0) \geq 0$ for all $\sigma \in W$, when $\psi$ is of the form $\varphi * \varphi^{\sim}$, where $\varphi^{\sim}=\overline{\varphi(-x)}$ and $\varphi \in \mathscr{S}(V)$. Here ${ }^{*}$ denotes the convolution operation.

If $\sigma \rightarrow \xi(\sigma)$ is a choice of unitary operators having properties (1), (3) and (4), then there exists a character $\eta$ of $F^{x} /\left(F^{x}\right)^{2}$ and a complex number $\varepsilon$ such that $\eta(-1)=\varepsilon^{2}$ and for $\sigma \in \Omega_{j}$,

$$
\xi(\sigma)=\eta(x(\sigma)) \varepsilon^{j} r(\sigma),
$$

where $x(\sigma)$ is defined by $x\left(p_{1} \tau_{S} p_{2}\right)=\operatorname{det}\left(p_{1} p_{2} \mid V^{*}\right)$ (see Lemma 5.1) and $\Omega_{j}$ is the $P$-double coset defined in Lemma 2.14 .

Proof. We use the notation of the lemmas and our first observation is that if the Haar measure $\mu_{\sigma}$ was chosen, then $\mu_{p_{1} \sigma p_{2}}$ can be defined as follows:

$$
\mu_{p_{1} \sigma p_{2}}=\left|\operatorname{det}\left(p_{1} p_{2} \mid V^{*}\right)\right|^{-1 / 2} \bar{p}_{1} \cdot \mu_{\sigma} .
$$

To see this gives a consistent definition suppose $p_{1} \sigma p_{2}=p_{1}^{\prime} \sigma p_{2}^{\prime}$. Then $p \sigma p^{\prime}=\sigma$ where $p=p_{1}^{\prime-1} p_{1}, p^{\prime}=p_{2} p_{2}^{\prime-1}$. Now we have to show that

$$
\left|\operatorname{det}\left(p_{1} p_{2} \mid V^{*}\right)\right|^{-1 / 2} \bar{p}_{1} \cdot \mu_{\sigma}=\left|\operatorname{det}\left(p_{1}^{\prime} p_{2}^{\prime} \mid V^{*}\right)\right|^{-1 / 2} \bar{p}_{1}^{\prime} \cdot \mu_{\sigma}
$$


or

$$
(\bar{p}) \cdot \mu_{\sigma}=\left|\operatorname{det}\left(p p^{\prime} \mid V^{*}\right)\right|^{1 / 2} \mu_{\sigma}
$$

Now $\bar{p}$ leaves $M_{\sigma}$ invariant and so

$$
\bar{p} \cdot \mu_{\sigma}=\left|\operatorname{det}\left(\bar{p} \mid M_{\sigma}\right)\right| \mu_{\sigma} .
$$

The consistency Lemma 3.4 now shows that $\mu_{p_{1} \sigma p_{2}}$ is well defined by (3.10). It is thus sufficient to choose $\mu_{\sigma}$ for each double coset of $P$. Let

$$
\mu_{I}=\delta_{0}, \quad \mu_{\tau_{s}}=d_{V_{s}^{*}},
$$

where we have the isomorphism of $V_{S}^{*}$ with $V^{*} / V_{S^{\prime}}^{*}$, to identify $M_{\tau_{S}}$ with $V_{S}^{*}$. The Haar measures $d_{V_{S}^{*}}$ were defined in 3.1. With this choice of Haar measures $\mu_{\sigma}$, define

$$
r(\sigma) \varphi: x \rightarrow \int_{M_{\sigma}} f_{\sigma}\left(x+x^{*}\right) \varphi\left(x \cdot \alpha+x^{*} \gamma\right) \mu_{\sigma}\left(d \bar{x}^{*}\right) .
$$

Then from Lemma 3.3 it is clear that the properties (1), (2) and (3) hold. It is clear that $r\left(\tau_{S}\right)$ is the partial Fourier transform given by formula (3.9). Since the Fourier transform operator on $V$ is the tensor product of those corresponding to $V_{1}, V_{2}$, when $V=V_{1}+V_{2}$ it is easy to see that $r\left(\tau_{S_{1} \cup S_{2}}\right)=r\left(\tau_{S_{1}}\right) r\left(\tau_{S_{2}}\right)$ when $S_{1}$ and $S_{2}$ are disjoint. On the other hand since the Haar measures $d_{V_{s}}, d_{V_{s}^{*}}$ are dual to each other, it follows that

$$
r\left(\tau_{S}\right)^{2}=r\left(a_{S}\right)
$$

where $\tau_{S}^{2}=a_{S} \in P$. These two properties now imply (see Lemma 2.13 on the structure of $W$ ) that $r\left(\sigma_{1} \sigma_{2}\right)=r\left(\sigma_{1}\right) r\left(\sigma_{2}\right)$ for all $\sigma_{1}, \sigma_{2} \in W$. To verify the property (5), note

$$
r\left(a_{S_{1}} \tau_{S_{2}}\right) \psi \cdot(0)=r\left(a_{S_{1}}\right) r\left(\tau_{S_{2}}\right) \psi \cdot(0)=r\left(\tau_{S_{2}}\right) \psi \cdot(0)
$$

and so it is sufficient to verify for $\tau_{S}$. Let $\varphi_{1} \in \mathscr{S}\left(V_{S_{1}}\right), \varphi_{2} \in \mathscr{S}\left(V_{S_{2}}\right)$ and $\varphi=\varphi_{1} \otimes \varphi_{2}$. If $\psi=\varphi^{*} \varphi^{\sim}$ then

$$
r\left(\tau_{S}\right) \psi \cdot(0)=\left|\mathscr{F}_{S} \varphi_{1}\right|^{2} \cdot(0) \cdot \int_{V_{S^{\prime}}}\left|\varphi_{2}(y)\right|^{2} d_{V_{S}} y
$$

Thus we have shown $r(\sigma)$ has all the properties (1) to (5). To prove uniqueness suppose $\sigma \rightarrow \xi(\sigma)$ is a choice of unitary operators satisfying (1), (3) and (4). Let the constants $c(\sigma)$ be defined by $\xi(\sigma)=$ $c(\sigma) r(\sigma)$. Then $c\left(p_{1} \sigma p_{2}\right)=c\left(p_{1}\right) c(\sigma) c\left(p_{2}\right)$ for all $p_{1}, p_{2} \in P$ and $c\left(\sigma_{1} \sigma_{2}\right)=c\left(\sigma_{1}\right) c\left(\sigma_{2}\right)$ for all $\sigma_{1}, \sigma_{2} \in W$. In particular $c$ is a character of $P$. Since $\operatorname{char} F \neq 2$, it is not difficult to show that the commutator subgroup $[P, P]$ of $P$ is $=\left\{\sigma \in P \mid \operatorname{det}\left(p \mid V^{*}\right)=1\right\}$. 
Thus there exists a character $\eta$ of the multiplicative group $F^{x}$, such that $c(p)=\eta\left(\operatorname{det}\left(p \mid V^{*}\right)\right)$, for all $p \in P$. If $S_{1}, S_{2}$ are two subsets of $\{1,2, \ldots, n\}$ having the same number of elements, then there exists a permutation $\alpha$ of $\{1,2, \ldots, n\}$ such that $\alpha\left(S_{1}\right)=S_{2}$. If $p \in P$ is defined as $e_{i} \cdot p=e_{\alpha(i)}, e_{i}^{*} \cdot p=e_{\alpha(i)}^{*}$, then $\tau_{S_{2}}=p^{-1} \tau_{S_{1}} p$. Thus $c\left(\tau_{S_{1}}\right)=c\left(\tau_{S_{2}}\right)$, whenever $S_{1}, S_{2}$ have the same number of elements. Since $c(\cdot)$ on $W$ is a character of $W$, it follows that $c\left(\tau_{S}\right)=\varepsilon^{j}$, where $j=|S|$. Thus $\varepsilon^{2 j}=c\left(\tau_{S}^{2}\right)=\eta\left(\operatorname{det}\left(\tau_{S}^{2} \mid V^{*}\right)\right)=\eta\left((-1)^{j}\right)$, $j=|S|$. From this it follows that $\varepsilon^{2}=\eta(-1)$. Suppose $p_{1} \tau_{S} p_{2}=\tau_{S}$; then $c\left(p_{1}\right) c\left(p_{2}\right)=1$ or $\eta\left(\operatorname{det}\left(p_{1} p_{2} \mid V^{*}\right)\right)=1$. From Lemma 3.5, it follows that $\eta\left(a^{2}\right)=1$, where $a=\operatorname{det}\left(\bar{p}_{1} \mid M_{\tau_{s}}\right)$. By choosing $p_{1}$ to be a suitable diagonal matrix, it follows that $\eta\left(a^{2}\right)=1$ for all $a \in F^{x}$. This proves the formula (3.10). Conversely if $\xi(\sigma)$ is defined by (3.10), it is clear that $\xi$ has properties (1), (3) and (4), since all the above arguments are reversible. To prove uniqueness of $r(\cdot)$, suppose $\xi(\cdot)$ has all the properties (1) to (5). Then since $\xi$ is a homomorphism on $P, \eta$ is a trivial character of $F^{x}$. Thus $\varepsilon^{2}=1$ and $\xi(\sigma)=\varepsilon^{j} r(\sigma)$, for $\sigma \in \Omega_{j}$. Now the non-negativity condition (5) implies that $\varepsilon=1$. This completes the proof.

Definition 3.6. The map $\sigma \rightarrow r(\sigma)$ is called the standard SegalShale-Weil (projective) representation. Note its construction depends only on the character $\chi$ of $F$ and the symplectic basis $e_{1}, \ldots, e_{n}^{*}$ of $X$. Note that $r(\sigma)$, for $\sigma \in W$ is given by the partial Fourier transform formula (3.9).

Good behaviour of the Weil representation for direct sums of symplectic vector spaces has been noted from the beginning. In terms of the standard model $\sigma \rightarrow r(\sigma)$, this can be formalized as follows.

Proposition 3.7. For any arbitrary subset $S$ of $\{1,2, \ldots, n\}$ let $r_{S}(\cdot)$ denote the standard Weil representation of $\operatorname{Sp}(X)$ corresponding to the data $X_{S}=V_{S}+V_{S}^{*}$, the symplectic basis being $\left\{e_{j}, e_{j}^{*}, j \in S\right\}$. Let $S_{1}, \ldots, S_{m}$ be a partition of $\{1,2, \ldots, n\}$ and let $\sigma_{j} \in \operatorname{Sp}(X)$, $j=1,2, \ldots, m$. If

$$
\sigma=\operatorname{diag}\left(\sigma_{1}, \ldots, \sigma_{m}\right) \in \operatorname{Sp}(X)
$$

then

$$
r(\sigma)=r_{S_{1}}\left(\sigma_{1}\right) \otimes \cdots \otimes r_{S_{m}}\left(\sigma_{m}\right) .
$$

Proof. Let $W_{j}$ denote the finite subgroup of $\operatorname{Sp}(X)$ introduced in $\S 2.5$ for the symplectic basis $\left\{e_{j}, e_{j}^{*}: j \in S_{j}\right\}$ of $X_{S_{j}}$. then it is easy 
to see that

$$
W=\left\{\operatorname{diag}\left(\sigma_{1}, \ldots, \sigma_{m}\right) \mid \sigma_{j} \in W_{j} \text { for all } j\right\} .
$$

From the definition of Fourier transform it is clear that the statement of the proposition is true when $\sigma \in W$ and $\sigma_{j} \in W_{j}$ for all $j$. It is clear that the proposition is valid when $\sigma_{j} \in P_{V_{S_{j}}}$, for all $j$. The general case now follows from properties (3) and (4) of Theorem 3.5, valid for each of the $r_{S}(\cdot)$.

4. Calculation of the multiplier. For the standard Weil representation $\sigma \rightarrow r(\sigma)$ introduced earlier, let $c\left(\sigma_{1}, \sigma_{2}\right)$ denote the multiplier, i.e. $c\left(\sigma_{1}, \sigma_{2}\right)$ is a complex number of absolute value one, defined by the relation

$$
r\left(\sigma_{1}\right) r\left(\sigma_{2}\right)=c\left(\sigma_{1}, \sigma_{2}\right) r\left(\sigma_{1} \sigma_{2}\right) .
$$

The following theorem gives an explicit formula for the multiplier in terms of the Leray invariant constructed in $\S 2$. The reader should note that the crucial computation (the part (4) in Theorem 4.1 below) is carried out already in Weil ([16], see Theorem 3, p. 163). For the definition and various properties of the Weil index of the character of second degree see the Appendix.

THEOREM 4.1. The multiplier $c\left(\sigma_{1}, \sigma_{2}\right)$ can be explicitly computed from the following properties:

(1) $c\left(p_{1} \sigma_{1} p, p^{-1} \sigma_{2} p_{2}\right)=c\left(\sigma_{1}, \sigma_{2}\right)$ for all $p, p_{1}, p_{2} \in P$ and $\sigma_{1}$, $\sigma_{2}$ arbitrary.

(2) $c\left(\sigma_{1}, \sigma_{2}\right)=1$ if $\sigma_{1}, \sigma_{2} \in W$.

(3) If $S_{1}, S_{2}, \ldots, S_{m}$ is a partition of $\{1,2, \ldots, n\}$ and $c_{S}(\cdot, \cdot)$ denotes the multiplier of the standard Weil representation $r_{S}$, then

$$
c\left(\sigma, \sigma^{\prime}\right)=\prod c_{S}\left(\sigma_{j}, \sigma_{j}^{\prime}\right)
$$

where $\sigma=\operatorname{diag}\left(\sigma_{1}, \ldots, \sigma_{m}\right), \sigma^{\prime}=\operatorname{diag}\left(\sigma_{1}^{\prime}, \ldots, \sigma_{m}^{\prime}\right)$.

(4) $c\left(\tau u_{\rho}, \tau\right)=$ Weil index of $\chi\left(\frac{1}{2}\langle x, x \cdot \rho\rangle\right)=\gamma\left(f_{u_{\rho}}\right)$.

(5) In general for any $\sigma_{1}, \sigma_{2} \in \operatorname{Sp}(X)$,

$$
c\left(\sigma_{1}, \sigma_{2}\right)=\text { Weil index of } \chi\left(\frac{1}{2}\langle x, x \cdot \rho\rangle\right)
$$

where the isometry class of $\rho$ is given by the Leray invariant $q\left(V^{*}, V^{*} \sigma_{2}^{-1}, V^{*} \sigma_{1}\right)$.

Proof. The property $r\left(p_{1} \sigma p_{2}\right)=r\left(p_{1}\right) r(\sigma) r\left(p_{2}\right)$ gives $c\left(p_{1} \sigma_{1}, \sigma_{2} p_{2}\right)$ $=c\left(\sigma_{1}, \sigma_{2}\right)$ and $c\left(\sigma_{1} p, p^{-1} \sigma_{2}\right)=c\left(\sigma_{1}, \sigma_{2}\right)$. This proves (1). Since 
$r(\cdot)$ restricted to $W$ is a representation (see Theorem 3.6 for the defining properties of $r(\cdot))$, it follows that $c\left(\sigma_{1}, \sigma_{2}\right)=1$ for all $\sigma_{1}, \sigma_{2} \in W$. The statement (3) follows from the tensor product property of $r(\cdot)$ stated in Proposition 3.8. The computation of $c\left(\tau u_{\rho}, \tau\right)$ is carried out in Weil (Theorem 3,p. 164). Actually since the formulas for $r(\tau), r\left(\tau u_{\rho}\right)$ and $r\left(\tau u_{\rho} \tau\right)$ are explicitly known the identity $r(\tau) r\left(u_{\rho}\right) r(\tau)=c\left(\tau u_{\rho}, \tau\right) r\left(\tau u_{\rho} \tau\right)$ leads quickly to a Fourier transform relation-which is the defining property of the Weil index. (See the Appendix, Theorem A.1.) Now suppose $\sigma_{1}, \sigma_{2}$ are arbitrary. Then by Theorem $2, \sigma_{1}=p_{1} \kappa_{1} p^{-1}, \sigma_{2}=p \kappa_{2} p_{2}$ where

$$
\kappa_{1}=\operatorname{diag}\left(\tau_{S_{1}}, \tau_{S} u_{\rho}\right), \quad \kappa_{2}=\operatorname{diag}\left(\tau_{S_{2}}, \tau_{S}\right) .
$$

Here the decomposition of $X$ is $=X_{S^{\prime}}+X_{S}, S^{\prime}$ being the complement of $S, S_{1} \subset S^{\prime}, S_{2} \subset S^{\prime}$. Also $\rho$ is nondegenerate on $V_{S}$ and the isometry class of $\rho$ is that of the Leray invariant $q\left(V^{*}, V^{*} \sigma_{2}^{-1}, V^{*} \sigma_{1}\right)$. It then follows that

$$
\begin{aligned}
c\left(\sigma_{1}, \sigma_{2}\right) & =c\left(\kappa_{1}, \kappa_{2}\right)=c_{S^{\prime}}\left(\tau_{S_{1}}, \tau_{S_{2}}\right) c_{S}\left(\tau u_{\rho}, \tau\right) \\
& =c_{S}\left(\tau u_{\rho}, \tau\right)=\text { Weil index of } \chi\left(\frac{1}{2}\langle x, x \cdot \rho\rangle\right) .
\end{aligned}
$$

This completes the proof.

Corollary 4.2. For any $\sigma, c\left(\sigma, \sigma^{-1}\right)=1$.

Proof. In this case the isometry class $q\left(V^{*}, V^{*} \sigma, V^{*} \sigma\right)$ is the trivial class. Thus $c\left(\sigma, \sigma^{-1}\right)=1$ for all $\sigma$.

Corollary 4.3. Suppose $\operatorname{dim} X=2$ and

$$
\sigma_{j}=\left[\begin{array}{cc}
a_{j} & b_{j} \\
c_{j} & d_{j}
\end{array}\right], \quad j=1,2
$$

Then

$$
c\left(\sigma_{1}, \sigma_{2}\right)=\left\{\begin{array}{l}
1 \quad \text { if } \sigma_{1}, \sigma_{2} \text { or } \sigma_{1} \sigma_{2} \in P, \\
\gamma_{F}\left(\frac{1}{2} c_{1} c_{2}\left(c_{1} a_{2}+d_{1} c_{2}\right) \chi\right) \text { otherwise. }
\end{array}\right.
$$

Here we have used the notation from the appendix, $\gamma_{F}(\eta)$ denoting the Weil index of $x \rightarrow \eta\left(x^{2}\right)$.

Proof. The formula for $c\left(\sigma_{1}, \sigma_{2}\right)$ follows from the calculation of the Leray invariant given in $\S 2.6$. The reader may note there are a large number of papers on the Weil representation of $\operatorname{SL}(2, F)$-see Gelbart [4] and also the references cited there. 
5. Normalization. It is known that the projective representation $r(\sigma)$ defines an ordinary representation for the two-fold cover of the group $\operatorname{Sp}(X)$. This is equivalent to the statement that one can find normalizing constants $m(\sigma)$, so that the multiplier for $r^{\sim}(\sigma)=$ $m(\sigma) r(\sigma)$ is \pm 1 valued. The object of this section is to construct such a normalization and also compute the multiplier explicitly. The reader may note that previously the existence of such a representation was deduced by an indirect argument (see Weil [16], §§42-43). An explicit formula for the multiplier given here agrees with that given by Kubota for the case $\operatorname{SL}(2, F)$. We begin with some preparation.

LEMMA 5.1. There exists a unique map $\sigma \rightarrow x(\sigma)$ of $\operatorname{Sp}(X)$ into $F^{x} /\left(F^{x}\right)^{2}$ such that the following properties hold:

(i) $x\left(p_{1} \sigma p_{2}\right)=x\left(p_{1}\right) x(\sigma) x\left(p_{2}\right)$, for all $p_{1}, p_{2} \in P$.

(ii) $x\left(\tau_{S}\right)=1$ for all subsets $S \subset\{1,2, \ldots, n\}$.

(iii) $x(p)=\operatorname{det}\left(p \mid V^{*}\right)\left(F^{x}\right)^{2}$, for all $p \in P$.

Moreover such a function is uniquely defined by

$$
x\left(p_{1} \tau_{S} p_{2}\right)=\operatorname{det}\left(p_{1} p_{2} \mid V^{*}\right)\left(F^{x}\right)^{2} .
$$

Proof. It is only necessary to show that if $S_{1}, S_{2}$ are two subsets and $p_{1} \tau_{S_{1}} p_{2}=p_{1}^{\prime} \tau_{S_{2}} p_{2}^{\prime}$, then $\operatorname{det}\left(p_{1} p_{2} \mid V^{*}\right)=\left(\operatorname{det} p_{1}^{\prime} p_{2}^{\prime} \mid V^{*}\right)\left(F^{x}\right)^{2}$. Now $\tau_{S_{1}}, \tau_{S_{2}}$ determine the same double coset if and only if $S_{1}, S_{2}$ have the same number of elements. Then there exists a permutation $\xi$ of the indices $\{1,2, \ldots, n\}$ such $\xi\left(S_{1}\right)=S_{2}$. If $a$ is the element of $P$ defined by $e_{i} \cdot a=e_{\xi(i)}, e_{i}^{*} \cdot a=e_{\xi(i)}^{*}$, then it is easy to check that $\tau_{S_{2}}=a^{-1} \cdot \tau_{S_{1}} \cdot a$. Thus $p_{1} \tau_{S_{1}} p_{2}=p_{1}^{\prime} a^{-1} \cdot \tau_{S_{1}} \cdot a \cdot p_{2}^{\prime}$. The lemma now follows from the consistency of Lemma 3.4.

Definition 5.2. Define the normalizing constants

$$
m(\sigma)=\gamma_{F}\left(x(\sigma), \frac{1}{2} \chi\right)^{-1}\left\{\gamma_{F}\left(\frac{1}{2} \chi\right)\right\}^{-j}
$$

for $\sigma \in \Omega_{j}=P \tau_{S} P$, with $j=|S|$. The quantities $\gamma_{F}(a, \eta), \gamma_{F}(\eta)$, for $a \in F^{x}$, and $\eta$ a character of $(F,+)$ are defined in the Appendix, $\S$ A.3.

Now define $r^{\sim}(\sigma)=m(\sigma) r(\sigma)$ and let $c^{\sim}(\cdot, \cdot)$ be the corresponding multiplier, i.e.

$$
r^{\sim}\left(\sigma_{1}\right) r^{\sim}\left(\sigma_{2}\right)=c^{\sim}\left(\sigma_{1}, \sigma_{2}\right) r^{\sim}\left(\sigma_{1} \sigma_{2}\right)
$$

Then it follows that

$$
c^{\sim}\left(\sigma_{1}, \sigma_{2}\right)=m\left(\sigma_{1}\right) m\left(\sigma_{2}\right)\left\{m\left(\sigma_{1} \sigma_{2}\right)\right\}^{-1} c\left(\sigma_{1}, \sigma_{2}\right) .
$$


THEOREM 5.3. The normalized projective representation $r^{\sim}(\cdot)$ is metaplectic, i.e. the corresponding multiplier is \pm 1 valued. In fact we have the explicit formula

$$
\begin{aligned}
c^{\sim}\left(\sigma_{1}, \sigma_{2}\right)= & \left(x\left(\sigma_{1}\right), x\left(\sigma_{2}\right)\right)_{F}\left(-x\left(\sigma_{1}\right) x\left(\sigma_{2}\right), x\left(\sigma_{1} \sigma_{2}\right)\right)_{F} \\
& \times\left((-1)^{l}, \operatorname{det} \rho\right)_{F}\left\{(-1,-1)_{F}\right\}^{l(l+1) / 2} h_{F}(\rho)
\end{aligned}
$$

where $\rho$ is the Leray invariant $-q\left(V^{*}, V^{*} \sigma_{1}, V^{*} \sigma_{2}^{-1}\right)$ (see $\S 2.3$ ), $h_{F}(\rho)$ is the Hasse invariant (see Appendix $\S \mathrm{A} .3$ ) and $2 l=j_{1}+j_{2}-j-$ $\operatorname{dim} \rho$, where $\sigma_{1} \in \Omega_{j_{1}}, \sigma_{2} \in \Omega_{j_{2}}, \sigma_{1} \sigma_{2} \in \Omega_{j}$.

Proof. First we compute (here $\eta=\frac{1}{2} \chi$ )

$$
\begin{aligned}
& m\left(\sigma_{1}\right) m\left(\sigma_{2}\right)\left\{m\left(\sigma_{1} \sigma_{2}\right)\right\}^{-1} \\
& =\left\{\gamma_{F}\left(x\left(\sigma_{1}\right), \eta\right) \gamma_{F}\left(x\left(\sigma_{2}\right), \eta\right)\right\}^{-1} \gamma_{F}\left(x\left(\sigma_{1} \sigma_{2}\right), \eta\right)\left\{\gamma_{F}(\eta)\right\}^{j-j_{1}-j_{2}}
\end{aligned}
$$

Now

$$
\begin{aligned}
& \gamma_{F}\left(x\left(\sigma_{1}\right), \eta\right) \gamma_{F}\left(x\left(\sigma_{2}\right), \eta\right) \\
& \quad=\left(x\left(\sigma_{1}\right), x\left(\sigma_{2}\right)\right)_{F} \gamma_{F}\left(x\left(\sigma_{1}\right) x\left(\sigma_{2}\right), \eta\right) .
\end{aligned}
$$

Now from Theorem 2.16 (of $\S 2.5$ ) we have

$$
\sigma_{1}=p_{1} \kappa_{1} p^{-1}, \quad \sigma_{2}=p \kappa_{2} p_{2}
$$

where

$$
\kappa_{1}=\operatorname{diag}\left(\tau_{S_{1}}, \tau_{S} u_{\rho}\right), \quad \kappa_{2}=\operatorname{diag}\left(\tau_{S_{2}}, \tau_{S}\right)
$$

and $j_{1}=|S|+\left|S_{1}\right|, j_{2}=|S|+\left|S_{2}\right|, j=|S|+\left|S_{1}\right|+\left|S_{2}\right|-2\left|S_{1} \cap S_{2}\right|$ and $\operatorname{dim} \rho=|S|$. Thus $l=\left|S_{1} \cap S_{2}\right|$. From the definition of $x(\cdot)$, it is clear that $x\left(\kappa_{1}\right)=1, x\left(\kappa_{2}\right)=1$, and

$$
\begin{gathered}
x\left(\sigma_{1}\right)=x\left(p_{1}\right) x(p), \quad x\left(\sigma_{2}\right)=x\left(p_{2}\right) x(p), \\
x\left(\sigma_{1} \sigma_{2}\right)=x\left(p_{1}\right) x\left(p_{2}\right) x\left(\kappa_{1} \kappa_{2}\right)=x\left(\sigma_{1}\right) x\left(\sigma_{2}\right) x\left(\kappa_{1} \kappa_{2}\right) .
\end{gathered}
$$

Thus

$$
\begin{aligned}
& \left\{\gamma_{F}\left(x\left(\sigma_{1}\right) x\left(\sigma_{2}\right), \eta\right)\right\}^{-1} \gamma_{F}\left(x\left(\sigma_{1} \sigma_{2}\right), \eta\right) \\
& \quad=\left(-x\left(\sigma_{1}\right) x\left(\sigma_{2}\right), x\left(\sigma_{1} \sigma_{2}\right)\right)\left\{\gamma_{F}\left(x\left(\kappa_{1} \kappa_{2}\right), \eta\right)\right\}^{-1} .
\end{aligned}
$$

Next $x\left(\kappa_{1} \kappa_{2}\right)=x\left(\tau_{S_{1}} \tau_{S_{2}}\right) x\left(\tau_{S} u_{\rho} \tau_{S}\right)=(-1)^{l} \operatorname{det} \rho$. That $\operatorname{det} \rho=$ $x\left(\tau_{S} u_{\rho} \tau_{S}\right)$ follows from the decomposition

$$
\tau u_{\rho} \tau=\left[\begin{array}{cc}
* & * \\
0 & \rho
\end{array}\right]\left[\begin{array}{cc}
0 & -I \\
I & 0
\end{array}\right]\left[\begin{array}{ll}
I & * \\
0 & I
\end{array}\right]
$$


and similar decompositions when $\tau$ is replaced by $\tau_{S}$. Thus

$$
\begin{aligned}
& \gamma_{F}\left(x\left(g k_{1} \kappa_{2}\right), \eta\right)=\gamma_{F}\left((-1)^{l} \operatorname{det} \rho, \eta\right) \\
& \quad=\left((-1)^{l}, \operatorname{det} \rho\right) \gamma_{F}\left((-1)^{l}, \eta\right) \gamma_{F}(\operatorname{det} \rho, \eta) .
\end{aligned}
$$

Next

$$
\begin{aligned}
\gamma_{F}\left((-1)^{l}, \eta\right)^{-1} \gamma_{F}(\eta)^{2 l} & =\gamma_{F}\left((-1)^{l}, \eta\right)^{-1}\left\{\gamma_{F}(-1, \eta)\right\}^{-l} \\
& =\{(-1,-1)\}^{l(l+1) / 2}
\end{aligned}
$$

Here we have used Corollary A.5 of the Appendix. Note that $j-j_{1}-$ $j_{2}=-\operatorname{dim} \rho+2 l$. Thus we get from $(5.2)-(5.6)$ that

$$
\begin{aligned}
m\left(\sigma_{1}\right) m & \left(\sigma_{2}\right)\left\{m\left(\sigma_{1} \sigma_{2}\right)\right\}^{-1} \\
= & \left(x\left(\sigma_{1}\right), x\left(\sigma_{2}\right)\right)\left(-x\left(\sigma_{1}\right) x\left(\sigma_{2}\right), x\left(\sigma_{1} \sigma_{2}\right)\right) \\
& \times\left((-1)^{l}, \operatorname{det} \rho\right)\{(-1,-1)\}^{l(l+1) / 2} \\
& \times\left\{\gamma_{F}(\operatorname{det} \rho, \eta)\right\}^{-1}\left\{\gamma_{F}(\eta)\right\}^{-j^{\prime}}
\end{aligned}
$$

where $j^{\prime}=\operatorname{dim} \rho$. Finally using the formula for $c\left(\sigma_{1}, \sigma_{2}\right)=\gamma\left(f_{\rho}\right)$ (see Theorem 3.6) we have

$$
c\left(\sigma_{1}, \sigma_{2}\right)\left\{\gamma_{F}(\operatorname{det}, \rho, \eta)\right\}^{-1}\left\{\gamma_{F}(\eta)\right\}^{-n}=h_{F}(\rho)
$$

from the definition of the Hasse invariant (see $\S$ A.3 of the Appendix). The theorem now follows from (5.7) and (5.8).

COROllary 5.4. For any $\sigma \in \Omega_{j}$

$$
c^{\sim}\left(\sigma, \sigma^{-1}\right)=\left(x(\sigma),(-1)^{j} x(\sigma)\right)_{F}\left\{(-1,-1)_{F}\right\}^{j(j+1) / 2} .
$$

Proof. Let $\sigma \in \Omega_{j}$, then $\sigma=p_{1} \tau_{S} p_{2}$ with $|S|=j$. Then $\sigma^{-1}=$ $p_{2}^{-1} \tau_{S}^{-1} p_{1}^{-1}=p_{2}^{-1} \tau_{S}^{-2} \tau_{S} p_{1}^{-1}$. Thus

$$
x\left(\sigma^{-1}\right)=(-1)^{j} x(\sigma) .
$$

In this case the Leray invariant is trivial. Thus

$$
c^{\sim}\left(\sigma, \sigma^{-1}\right)=\left(x(\sigma), x\left(\sigma^{-1}\right)\right)\left(-x(\sigma) x\left(\sigma^{-1}\right), 1\right) \times\{(-1,1)\}^{j(j+1) / 2}
$$

and this simplifies to the statement of the lemma.

COROLlaRY 5.5. (1) $c^{\sim}\left(\tau_{S_{1}}, \tau_{S_{2}}\right)=\{(-1,-1)\}^{l(l+1) / 2}$ where $l=$ $\left|S_{1} \cap S_{2}\right|$.

(2) $c^{\sim}\left(p_{1}, p_{2}\right)=\left(x\left(p_{1}\right), x\left(p_{2}\right)\right)_{F}$, for all $p_{1}, p_{2} \in P$. 
(3)

(4) $c^{\sim}\left(\sigma_{1} p^{-1}, p \sigma_{2}\right)\left\{c^{\sim}\left(\sigma_{1}, \sigma_{2}\right)\right\}^{-1}=\left(x(p),-x\left(\sigma_{1}\right) x\left(\sigma_{2}\right)\right)$.

Proof. Note

$$
\begin{aligned}
m(p \sigma) & =(x(p), x(\sigma)) m(p) m(\sigma), \\
m(\sigma p) & =(x(\sigma), x(\sigma)) m(\sigma) m(p), \\
m\left(p^{-1}\right) & =m(p) \text { and } m(p)^{2}=(x(p),-1)_{F} .
\end{aligned}
$$

The results then follow by straightforward computation from (5.1).

CoRollary 5.6. For any subset $S \subset\{1,2, \ldots, n\}$, let $m_{S}(\cdot)$, $c_{S}(\cdot, \cdot)$ be defined analogously for $\operatorname{Sp}\left(X_{S}\right)$. Then if $S_{1}, S_{2}$ is a partition of $\{1,2, \ldots, n\}$, then

$$
\begin{aligned}
& c^{\sim}\left(\sigma, \sigma^{\prime}\right)\left\{\tilde{S}_{1_{1}}\left(\sigma_{1}, \sigma_{1}^{\prime}\right){\tilde{S_{2}}}_{2}\left(\sigma_{2}, \sigma_{2}^{\prime}\right)\right\}^{-1} \\
& \quad=\left(x_{S_{1}}\left(\sigma_{1}\right), x_{S_{2}}\left(\sigma_{2}\right)\right)\left(x_{S_{1}}\left(\sigma_{1}^{\prime}\right), x_{S_{2}}\left(\sigma_{2}^{\prime}\right)\right)\left(x_{S_{1}}\left(\sigma_{1} \sigma_{1}^{\prime}\right), x_{S_{2}}\left(\sigma_{2} \sigma_{2}^{\prime}\right)\right)
\end{aligned}
$$

where $\sigma_{j}, \sigma_{j}^{\prime} \in \operatorname{Sp}\left(X_{S_{j}}\right)$ and

$$
\sigma=\operatorname{diag}\left(\sigma_{1}, \sigma_{2}\right), \quad \sigma^{\prime}=\operatorname{diag}\left(\sigma_{1}^{\prime}, \sigma_{2}^{\prime}\right) .
$$

Proof. It is easy to check that

$$
\begin{gathered}
x(\sigma)=x_{S_{1}}\left(\sigma_{1}\right) x_{S_{2}}\left(\sigma_{2}\right), \\
m(\sigma)=\left(x_{S_{1}}\left(\sigma_{1}\right), x_{S_{2}}\left(\sigma_{2}\right)\right) m_{S_{1}}\left(\sigma_{1}\right) m_{S_{2}}\left(\sigma_{2}\right) .
\end{gathered}
$$

The result then follows easily from this and the fact that $c\left(\sigma, \sigma^{\prime}\right)=$ $c_{S_{1}}\left(\sigma_{1}, \sigma_{1}^{\prime}\right) c_{S_{2}}\left(\sigma_{2}, \sigma_{2}^{\prime}\right)$ (see Theorem 4.1).

The following corollary is well known.

COROLlary 5.7. When $F$ is a finite field or the field of complex numbers, $c^{\sim}\left(\sigma_{1}, \sigma_{2}\right)=1$ for all $\sigma_{1}, \sigma_{2}$. In particular $r^{\sim}(\sigma)$ is a representation in this case.

Proof. This follows from the observation that both the Hilbert symbol and the Hasse invariant are equal to 1 in this case. 
REMARK. We note here that when $\operatorname{dim} X=2$, we have

$$
c^{\sim}\left(\sigma_{1}, \sigma_{2}\right)=\left(x\left(\sigma_{1}\right), x\left(\sigma_{2}\right)\right)_{F}\left(-x\left(\sigma_{1}\right) x\left(\sigma_{2}\right), x\left(\sigma_{1} \sigma_{2}\right)\right)_{F} .
$$

In fact in this case $n=1$, so the Hasse invariant is always one and when $\operatorname{dim} \rho \neq 0, l=0$. Also the definition of $x(\sigma)$ reduces to

$$
x:\left(\begin{array}{ll}
a & b \\
c & d
\end{array}\right) \rightarrow \begin{cases}d\left(F^{x}\right)^{2} & \text { when } c=0, \\
c\left(F^{x}\right)^{2} & \text { when } c \neq 0 .\end{cases}
$$

Thus the multiplier agrees with that given by Kubota. In this connection see Kubota [7], or the exposition in Gelbart [4].

Finally we end this with a proposition due to Weil ([16], see $\S 44)$. The proof given will be based on formula (3.10) of Theorem 3.5 and is different from that of Weil.

Proposition 5.8. The projective representation $\sigma \rightarrow r(\sigma)$ is equivalent to an ordinary representation (or the cohomology class of the multiplier $c(\cdot, \cdot)$ is trivial) if and only if $F$ is either a finite field or is the field of complex numbers.

Proof. In view of Corollary 5.5 we have only to show the necessity of the condition. If the representation is equivalent to an ordinary representation there exist constants $c(\sigma)$ such that if $\xi(\sigma)=c(\sigma) r(\sigma)$, then $\xi\left(\sigma_{1} \sigma_{2}\right)=\xi\left(\sigma_{1}\right) \xi\left(\sigma_{2}\right)$ for all $\sigma_{1}, \sigma_{2}$. From Theorem 3.6, it follows that $c(\sigma)=\eta(x(\sigma)) \varepsilon^{j}$ for $\sigma \in \Omega_{j}$, where $\varepsilon^{2}=\eta(-1)$ and $\eta$ is a character of $F^{x} /\left(F^{x}\right)^{2}$. Since $\xi$ is a homomorphism it follows that

$$
\eta\left(x\left(\sigma_{1}\right)\right) \varepsilon^{j_{1}} \eta\left(x\left(\sigma_{2}\right)\right) \varepsilon^{j_{2}} c\left(\sigma_{1}, \sigma_{2}\right)=\eta\left(x\left(\sigma_{1} \sigma_{2}\right)\right) \varepsilon^{j}
$$

where $\sigma_{1} \in \Omega_{j_{1}}, \sigma_{2} \in \Omega_{j_{2}}, \sigma_{1} \sigma_{2} \in \Omega_{j}$. Here $c\left(\sigma_{1}, \sigma_{2}\right)$ is the multiplier of $r(\cdot)$. Let $\sigma_{1}=\tau_{S} u_{\rho}, \sigma_{2}=\tau_{S}$; then it follows that

$$
c\left(\tau_{S} u_{\rho}, \tau_{S}\right)=\gamma\left(f_{\rho}\right)=\eta(\operatorname{det} \rho) \varepsilon^{-j}
$$

where $j=|S|$ (see Theorem 3.6 for $\left.c\left(\tau_{S} u_{\rho}, \tau_{S}\right)\right)$. Taking $j=1$, we get

$$
\gamma_{F}\left(\frac{1}{2} a \chi\right)=\varepsilon^{-1} \eta(a) .
$$

From this it follows that $\gamma_{F}\left(a, \frac{1}{2} \chi\right)=\eta(a)$ for all $a \in F^{x}$. Since $\eta$ is a character of $F^{x}$, it follows that $(a, b)_{F}=1$ for all $a, b \in F^{x}$. This can happen only if $F$ is either a finite field or, the field of complex numbers. 
Appendix. The Weil index of a character of second degree.

A.1. Let $G, G^{*}$ be locally compact abelian groups ad $\chi$ a nondegenerate bicharacter pairing of $G$ and $G^{*}$. Let $\mathscr{S}(G), \mathscr{S}\left(G^{*}\right)$ denote the Bruhat-Schwartz spaces of functions on $G, G^{*}$ respectively. Write

$$
\begin{gathered}
\mathscr{F} \varphi: x^{*} \rightarrow \int \chi\left(x, x^{*}\right) \varphi(x) d_{G} x, \\
\mathscr{F}^{*} \psi: x \rightarrow \int \chi\left(x, x^{*}\right) \psi\left(x^{*}\right) d_{G^{*}} x^{*}
\end{gathered}
$$

where $d_{G}, d_{G^{*}}$ denote Haar measures on $G, G^{*}$ respectively, and $\varphi \in \mathscr{S}(G), \psi \in \mathscr{S}\left(G^{*}\right)$. Then there exists a pairing of the Haar measures, called the Plancherel pairing, such that

$$
\mathscr{F}^{*} \mathscr{F} \varphi=\left(d_{G}, d_{G^{*}}\right)_{\chi} \cdot \varphi^{\circ}, \quad \mathscr{F F}^{*} \psi=\left(d_{G}, d_{G^{*}}\right)_{\chi} \cdot \psi^{\circ},
$$

where $\varphi^{\circ}(x)=\varphi(-x), \psi^{\circ}\left(x^{*}\right)=\psi\left(-x^{*}\right)$. The Haar measures $d_{G}$, $d_{G^{*}}$ are said to be dual to each other relative to the pairing $\chi$, if $\left(d_{G}, d_{G^{*}}\right)_{\chi}=1$. If $u$ is a tempered distribution on $G$, i.e. $u$ is a complex valued continuous linear functional on $\mathscr{S}(G)$, then its Fourier transform $\mathscr{F} u$ is a tempered distribution on $G^{*}$ defined as follows:

$$
\mathscr{F} u: \psi \rightarrow u\left(\mathscr{F}^{*} \psi\right) \text {. }
$$

Fourier transforms of tempered distributions on $G^{*}$ are denoted by $\mathscr{F}^{*}$. If $f$ is a function on $G, f d_{G}$ is the distribution $\varphi \rightarrow \int f \varphi d_{G}$.

A.2. A character of the second degree $f$ on $G$ is a continuous map of $G$ into $T$ such that $f(x+y)\{f(x) f(y)\}^{-1}$ is a bicharacter in $x$ and $y$. In particular there exists a continuous homomorphism $\rho=\rho(f)$ of $G$ into $G^{*}$ such that

$$
f(x+y)\{f(x) f(y)\}^{-1}=\chi(x, y \cdot \rho)=\chi(y, x \cdot \rho)
$$

for all $x, y \in G$. Then $f$ is said to be nondegenerate if $\rho$ is an isomorphism of $G$ with $G^{*}$. For the following theorem see Weil [16] (see also Cartier [3], for another exposition). Note the definition of the Fourier transform depends on the pairing $\chi$.

THEOREM A.1. Let $f$ be a nondegenerate character of the second degree on $G$, and let $\rho=\rho(f)$ be the associated symmetric homomorphism. Then there exists a complex constant $\gamma(f)$ of modulus one, such that ${ }^{3}$

$$
\mathscr{F}\left(f d_{G}\right)=\gamma(f)\left\{\left(d_{G}, \rho \cdot d_{G}\right)_{\chi}\right\}^{-1 / 2} f^{\prime} d_{G^{*}}
$$

\footnotetext{
${ }^{3}$ Here $\rho \cdot d_{G}$ is the measure on $G^{*}$ defined by the identity $\rho \cdot d_{G}(\varphi \circ \rho)=d_{G}(\varphi)$, for all $\varphi \in \mathscr{S}(G)$.
} 
where $f^{\prime}$ is a character of second degree on $G^{*}$ defined by the formula $f^{\prime}\left(x^{*}\right)=\left\{f\left(x^{*} \rho^{-1}\right)\right\}^{-1}$. The constant $\gamma(f)$ is independent of the pairing $\chi$ or the Haar measures used in its definition. We call $\gamma(f)$ the Weil index of $f$.

The next theorem summarizes elementary properties of the Weil index. They are deduced easily from the definition.

THeOREM A.2. (1) $\gamma(f \circ \alpha)=\gamma(f)$, for any continuous automorphism $\alpha$ of $G$.

(2) $\gamma(\bar{f})=\overline{\gamma(f)}, \bar{f}$ denoting the complex conjugate of $f$.

(3) If $G=G_{1} \times G_{2}$, and $f_{1}, f_{2}$ are nondegenerate characters of second degree on $G_{1}, G_{2}$ respectively and $f=f_{1} \times f_{2}$, then $\gamma(f)=$ $\gamma\left(f_{1}\right) \gamma\left(f_{2}\right)$.

(4) For any $x^{*} \in G^{*}$, let $x^{*} f$ denote the function $x \rightarrow \chi\left(x, x^{*}\right) f(x)$, then

$$
\gamma\left(x^{*} f\right)=\gamma(f) f^{-1}\left(x^{*} \rho^{-1}\right) .
$$

(5) If $G$ is a finite group, $\gamma(f)$ is a Gauss sum, i.e.

$$
\gamma(f)=|G|^{-1 / 2} \sum_{x \in G} f(x)
$$

where $|G|$ is the number of elements of $G$.

In the next theorem, the first part is due to Weil ([16], see Theorem 5 ), and he bases his proof of the quadratic reciprocity law on this theorem. The second part is the main technique by which evaluation of the Weil index is reduced to that of Gauss sums. This is implicit in Weil but explicitly stated and proved in Cartier [3].

Theorem A.3. Let $\Gamma$ be a closed subgroup of $G$ and $\Gamma_{*}$ its annihilator in $G$. Let $f$ be a nondegenerate character of second degree on $G$.

(1) If $f \mid \Gamma=1$ and $\Gamma \rho=\Gamma_{*}$, then $\gamma(f)=1$.

(2) If $f \mid \Gamma=1$, then $\Gamma \rho \subset \Gamma_{*}$ and the function $g(x+\Gamma)=f(x), x \in$ $\Gamma_{*} \rho^{-1}$ is a well-defined nondegenerate character of second degree on $H=\Gamma_{*} \rho^{-1} / \Gamma$, and the Weil index of $f$ is equal to the Weil index of $g$, i.e. $\gamma(f)=\gamma(g)$.

A.3. For the remainder of the Appendix, let $F$ be a self-dual locally compact field with char $F \neq 2$, i.e. $F$ is either a finite field or a local field. For the material of this part see Weil [16], Saito [12], Rallis and Schiffman [11]. 
Let $\eta$ be a nontrivial continuous character of $(F,+)$. For any $a \in F$, we write $a \eta$ for the character $a \eta: x \rightarrow \eta(a x)$. Define

$$
\begin{aligned}
\gamma_{F}(\eta) & =\text { Weil index of: } x \rightarrow \eta\left(x^{2}\right), \\
\gamma_{F}(a, \eta) & =\gamma_{F}(a \eta) / \gamma_{F}(\eta), \quad a \in F^{x} .
\end{aligned}
$$

The main theorem on the $\gamma_{F}(a, \eta)$ is the following (see Weil [16], p. 176).

THEOREM A.4. $\gamma_{F}\left(a c^{2}, \eta\right)=\gamma_{F}(a, \eta)$ and the function $a \rightarrow \gamma_{F}(a, \eta)$ is a character of second degree on $F^{x} /\left(F^{x}\right)$ and moreover

$$
\gamma_{F}(a b, \eta) \gamma_{F}(a, \eta)^{-1} \gamma_{F}(b, \eta)^{-1}=(a, b)_{F}
$$

where $(a, b)_{F}$ is the Hilbert symbol of $F$ i.e.

$$
(a, b)_{F}= \begin{cases}+1 & \text { if } a \text { is a norm in } F(\sqrt{b}), \\ -1 & \text { otherwise. }\end{cases}
$$

The following corollary is immediate.

CoROllaRY A.5. (1) $\gamma_{F}(a, c \eta)=(a, c)_{F} \gamma_{F}(a, \eta)$.

(2) $\gamma_{F}(-1, \eta)=\gamma_{F}(\eta)^{-2}$.

(3) $\left\{\gamma_{F}(a, \eta)\right\}^{2}=(-1, a)_{F}=(a, a)_{F}$.

(4) $\left\{\gamma_{F}(a, \eta)\right\}^{4}=1$ and $\left\{\gamma_{F}(\eta)\right\}^{8}=1$.

Explicit evaluation of $\gamma_{F}(a, \eta)$ will be given in the next section.

Definition A.6. Let $Q$ be a nondegenerate quadratic form of degree $n$ over $F$. Then the Hasse invariant $h_{F}(Q)$ is defined as follows:

$$
h_{F}(Q)=\gamma(\eta \circ Q)\left\{\gamma_{F}(\eta)\right\}^{-n}\left\{\gamma_{F}(\operatorname{det} Q, \eta)\right\}^{-1} \text {. }
$$

Here $\gamma(\eta \circ Q)$ is the Weil index of the character of second degree $x \rightarrow \eta(Q(x))$ and it will be shown below that the expression on the right is independent of $\eta$.

LemMA A.7. (1) If $n=1, h_{F}(Q)=1$.

(2) If $n=2$, and $Q=a_{1} x_{1}^{2}+a_{2} x_{2}^{2}, a_{1}, a_{2} \in F^{x}$, then $h_{F}(Q)=$ $\left(a_{1}, a_{2}\right)_{F}$.

(3) If $Q=Q_{1}+Q_{2}+\cdots+Q_{m}$, then

$$
h_{F}(Q)=\left\{\prod h_{F}\left(Q_{i}\right)\right\} \prod_{i<j}\left(\operatorname{det} Q_{i}, \operatorname{det} Q_{j}\right)_{F} .
$$


In particular

$$
h_{F}\left(\left\langle a_{1} x_{1}^{2}+\cdots+a_{n} x_{n}^{2}\right\rangle\right)=\prod_{i<j}\left(a_{i}, a_{j}\right)_{F} .
$$

Proof. The parts (1) and (2) are obvious. The part (3) is proved by induction on $m$ and using the Theorem A.2 (see [11]).

COROllary A.8. If $Q$ is hyperbolic of degree $2 \mathrm{~m}$, then

$$
h_{F}(Q)=\left\{(-1,-1)_{F}\right\}^{l}, \quad \text { where } l=m(m-1) / 2 \text {. }
$$

A.4. In this section we note some of the explicit evaluations that are known about $\gamma_{F}(\eta)$ and the Hasse invariant.

Proposition A.9. Suppose $F$ is a finite field of char $\neq 2$. Then (1)

$$
\gamma_{F}(a, \eta)=\left(\frac{a}{F}\right)= \begin{cases}+1 & \text { if } a \text { is a square, } \\ -1 & \text { otherwise. }\end{cases}
$$

(2) $a \rightarrow \gamma_{F}(a, \eta)$ is a homomorphism and $(a, b)_{F}=1$ for all $a, b \in F^{x}$.

(3) $h_{F}(Q)=1$ for any $Q$.

(4) If $\mathbb{F}_{p}$ is the prime subfield of $F$, and $[F: \mathbb{F}]=n$ and $\eta^{\prime}=\eta \circ \mathrm{tr}$, then

$$
\gamma_{F}\left(\eta^{\prime}\right)=\left\{\gamma_{\mathbb{F}_{p}}(\eta)\right\}^{n} \text {. }
$$

(5) If $\eta$ denotes the character $j \rightarrow \exp (2 \pi j \sqrt{-1} / p)$, then the Gauss sum

$$
\gamma_{\mathbb{F}_{p}}(\eta)= \begin{cases}1 & \text { if } p \equiv 1 \bmod 4 \\ \sqrt{-1} & \text { otherwise }\end{cases}
$$

Proof. These are all well-known results, see for instance Serre's book on arithmetic. The part (5) is a famous result of Gauss and Landau's book on elementary number theory has several proof of it. It may be of some interest to mention that another proof of it (actually for any odd integer $n$ ) can be given on the basis of (4) of Theorem A.2, Theorem A.3 and the evaluation of $\gamma_{R}(\eta)$ for reals.

Proposition A.10. (1) When $F$ is the field of complex numbers, $\gamma_{F}(\eta),(a, b)_{F}, h_{F}(Q)$ are all equal to 1 .

(2) Suppose $F$ is the field of real numbers. Then

$$
(a, b)_{F}= \begin{cases}-1 & \text { if } a<0, b<0, \\ +1 & \text { otherwise. }\end{cases}
$$


If $\eta(t)=\exp (2 \pi t \sqrt{-1})$, then

$$
\gamma_{F}(a \eta)=\eta((\operatorname{sign} a) / 8) .
$$

Also if $Q$ is a quadratic form of signature $(a, b), b$ being the number of negative eigenvalues, then

$$
h_{F}(Q)=(-1)^{m}, \quad \text { where } m=b(b-1) / 2 \text {. }
$$

For the rest of this section let $F$ be a non archimedean local field with char $F \neq 2, R$ the ring of integers of $F, \pi$ a generator of the maximal ideal of $R, \bar{F}$ the residue field of $F$. Let $\eta$ be a nontrivial character of $(F,+)$ and let ord $\eta$ denote the largest integer $m$ such that $\eta=1$ on $\pi^{-m} R$. Let $\alpha(\eta)$ denote the parity of ord $\eta$, i.e. $\alpha(\eta)=0$ or 1 according as ord $\eta$ is even or odd.

Proposition A.11. Suppose char $\bar{F} \neq 2$. Let

$$
\bar{\eta}: x+\pi R \rightarrow \eta\left(\pi^{-m-1} x\right) \text {. }
$$

Then $\bar{\eta}$ is a nontrivial character of $\bar{F}$ and

$$
\gamma_{F}(\eta)=\left\{\gamma_{\bar{F}}(\bar{\eta})\right\}^{\alpha(\eta)} .
$$

Moreover

$$
\gamma_{F}(a, \eta)=\left\{\left(\frac{\bar{u}}{\bar{F}}\right) \gamma_{\bar{F}}(\bar{\eta})\right\}^{\alpha(a)}
$$

where $a=\pi^{\operatorname{ord} a} \cdot u, u$ being a unit of $R$.

Proof. These are all known. All the other formulas can be deduced once the relation between $\gamma_{F}(\eta)$ and $\gamma_{\bar{F}}(\bar{\eta})$ is established. This can be done as follows. Let $m=$ ord $\eta$. Let $f(x)=\eta\left(x^{2}\right), \Gamma=\pi^{-r} R$, where $r$ is the integral part of $m$. Then $\Gamma_{*}=\{x \in F \mid \eta(x \Gamma)=1\}$ $=\pi^{r-m} R$. Now $\rho(f): x \rightarrow 2 x$, relative to the pairing of $F$ with itself defined by $\eta$. Since 2 is a unit in $R$, the subgroup $H=$ $\Gamma_{*} \rho^{-1} / \Gamma=\pi^{r-m} R / \pi^{-r} R$. Thus $H$ is trivial and $\gamma(f)=1$ when ord $\eta$ is even (see Theorem A.3). Now suppose $m=$ ord $\eta$ is odd. Then $H=\pi^{r-m}(R / R)=\pi^{r-m} \bar{F}$. Now let $\bar{\eta}: x \rightarrow \pi R \rightarrow \eta\left(\pi^{-m-1} x\right)$. Then $\bar{\eta}$ is a nontrivial character of $\bar{F}$ and $\gamma(g)=\gamma_{\bar{F}}(\bar{\eta})$. In this connection see also Saito [12] and Serre's book.

Finally we assume that $F$ is a dyadic local field of char $\neq 2$. For any nontrivial character $\eta$ on $F$ let $\alpha(\eta)$ denote the parity of ord $\eta$ and $\beta=\beta(\eta)=e+\alpha(\eta)$. Here $e=\operatorname{ord}_{\pi} 2$. Let $A_{\beta}$ denote the ring $R / \pi^{\beta} R$. Let $\bar{\eta}: x+\pi^{\beta} R \rightarrow \eta\left(\pi^{-\beta-m} x\right)$, where $m=$ ord $\eta$. 
Then $\bar{\eta}$ is a primitive character of $A_{\beta}$, i.e. $\bar{x}, \bar{y} \rightarrow \bar{\eta}(\overline{x y})$ defines a nondegenerate pairing of $A_{\beta}$ with itself. Let

$$
g_{\eta}\left(x+\pi^{\beta} R\right)=\eta\left(\pi^{-\beta-m-e} x^{2}\right) .
$$

Then $g_{\eta}$ is a character of second degree, satisfying

$$
g_{\eta}(\bar{x}+\bar{y}) g_{\eta}(\bar{x})^{-1} g_{\eta}(\bar{y})^{-1}=\bar{\eta}(\overline{x y}) \text {. }
$$

Now we apply Theorem A.4 to $f_{\eta}(x)=\eta\left(x^{2}\right)$ with $\Gamma=\pi^{-r} R$, where $r$ is the integral part of $m / 2$. One gets that $\gamma\left(f_{\eta}\right)=\gamma\left(g_{\eta}\right)$. Thus

$$
\gamma_{F}(\eta)=\left|A_{\beta}\right|^{-1 / 2} \sum_{\bar{x} \in A} \eta\left(\pi^{-\beta-m-e} x^{2}\right) .
$$

It is not clear whether this Gaussian sum can be simplified further, in general. However this is possible when $F=Q_{2}$.

Proposition A.12. Let $F=Q_{2}$ and let $\eta$ be a character with ord $\eta=0$. Then

$$
\gamma_{F}(\eta)=2^{-1 / 2}\left(1+\eta\left(\frac{1}{4}\right)\right)
$$

and

$$
\gamma_{F}(a, \eta)= \begin{cases}\left\{\eta\left(-\frac{1}{4}\right)\right\}^{\varepsilon_{1}(u)} & \text { if } a=u, \\ (-1)^{\omega(u)}\left\{\eta\left(-\frac{1}{4}\right)\right\}^{\varepsilon_{1}}(u) & \text { if } a=2 u\end{cases}
$$

where $\varepsilon_{1}, \varepsilon_{2}$ are homomorphisms of $U$ defined by $u=1+2 \varepsilon_{1}+$ $4 \varepsilon_{2} \bmod 8$ and $\omega(u)=\varepsilon_{1}+\varepsilon_{2}$.

\section{REFERENCES}

[1] N. Bourbaki, Algèbres, Chapter 9, Hermann, Paris, 1959.

[2] F. Bruhat, Distributions sur un groupe localement compact et applications, Bull. Soc. Math. de France, 89 (1961), 43-75.

[3] P. Cartier, Über einige Integral formeln in der theorie der quadratische formen, Math. Z., 84 (1964), 93-100.

[4] S. S. Gelbart, Weil's Representation and the Spectrum of the Metaplectic Group, Lecture Notes in Math., vol. 530, Springer-Verlag, New York, 1976.

[5] Paul Gérardin, Weil representations associated to finite fields, J. Algebra, 46 (1977), 54-102.

[6] V. Guillemin and S. Sternberg, Geometric asymptotics, Math. Surveys no. 14, Amer. Math. Soc., Providence, RI, 1977.

[7] T. Kubota, Topological covering of $S L(2)$ over a local field, J. Math. Soc. Japan, 19 (1967), 114-121.

[8] Jean Leray, Complèment à la théorie d'Arnold de l'indice de Maslov, Symposia Math., XIV (1974), 33-53.

[9] G. Lion, Integrales Interlacement sur des Groupes de Lie Nilpotents et Indices de Maslov, Lecture Notes in Math., vol. 587, Springer-Verlag, New York, 1977, pp. $160-177$. 
[10] G. W. Mackey, The theory of unitary representations, Chicago Lectures in Math., Chicago Univ. Press, 1976.

[11] S. Rallis and G. Schiffman, Distribution Invariant par le Groupe Orthogonal, Lecture Notes in Math., vol. 497, Springer-Verlag, New York, 1975, pp. 494643.

[12] M. Saito, Representations unitaires des groupes symplectiques, J. Math. Soc. Japan, 24 (1972), 232-252.

[13] I. E. Segal, Transforms for operators and symplectic automorphisms over a locally compact abelian group, Math. Scand., 12-13 (1963), 31-43.

[14] J. P. Serre, A Course in Arithmetic, Springer, New York, 1973.

[15] D. Shale, Linear symmetries of free boson fields, Trans. Amer. Math. Soc., 103 (1962), 149-167.

[16] A. Weil, Sur certaines groupes d'opérateurs unitaires, Acta Math., 11 (1964), 143-211.

[17] N. R. Wallach, Symplectic Geometry and Fourier Analysis, Math. Sci. Press, Brookline, Massachusetts, 1977.

[18] C. C. Moore, Group extensions of p-adic and adelic linear groups, I.H. E. S., 1968.

[19] G. B. Folland, Harmonic analysis in phase space, Ann. of Math. Studies, Princeton University Press, 1989.

[20] G. Lion and M. Vergne, The Weil representation Maslov index and Theta series, Progress in Math., vol. 6, Birkhäuser, Boston, 1980.

[21] C. Moeglin, M. F. Vigneras, and J. L. Waldspurger, Correspondences de Howe sur un Corps p-adique, Lecture Notes in Math., vol. 1291, Springer-Verlag, Berlin, 1987.

Received May 13, 1991 and in revised form December 10, 1991. Research partially supported by an NSF grant.

UNIVERSITY OF ILLINOIS

URBANA, IL 61801 



\title{
PACIFIC JOURNAL OF MATHEMATICS
}

\author{
Founded by
}

E. F. BECKENBACH (1906-1982) F. WoLF (1904-1989)

\section{EDITORS}

$\begin{array}{ll}\begin{array}{l}\text { V. S. VARADARAJAN } \\ \text { (Managing Editor) }\end{array} & \begin{array}{l}\text { NiCHOLAS ERCOLANI } \\ \text { University of Arizona }\end{array} \\ \text { University of California } & \text { Tucson, AZ 85721 } \\ \text { Los Angeles, CA 90024-1555 } & \text { ercolani@math.arizona.edu } \\ \text { vsv@math.ucla.edu } & \text { R. FinN } \\ \text { HERBERT CLEMENS } & \text { Stanford University } \\ \text { University of Utah } & \text { Stanford, CA 94305 } \\ \text { Salt Lake City, UT 84112 } & \text { finn@gauss.stanford.edu } \\ \text { clemens@math.utah.edu } & \text { VAUGHAN F. R. JoNEs } \\ \text { F. MichAEL CHRIsT } & \text { University of California } \\ \text { University of California } & \text { Berkeley, CA 94720 } \\ \text { Los Angeles, CA 90024-1555 } & \text { vfr@math.berkeley.edu } \\ \text { christ@math.ucla.edu } & \text { STEVEN KERCKHoFF } \\ \text { THomas ENRIGHT } & \text { Stanford University } \\ \text { University of California, San Diego } & \text { Stanford, CA 94305 } \\ \text { La Jolla, CA 92093 } & \text { spk@gauss.stanford.edu } \\ \text { tenright@ucsd.edu } & \end{array}$

\author{
C. C. MOORE \\ University of California \\ Berkeley, CA 94720
}

MaRTin ScharlemanN

University of California

Santa Barbara, CA 93106

mgscharl@henri.ucsb.edu

\author{
HAROLD STARK \\ University of California, San Diego \\ La Jolla, CA 92093
}

\begin{tabular}{ll}
\multicolumn{1}{c}{ SUPPORTING } & INSTITUTIONS \\
UNIVERSITY OF ARIZONA & UNIVERSITY OF OREGON \\
UNIVERSITY OF BRITISH COLUMBIA & UNIVERSITY OF SOUTHERN CALIFORNIA \\
CALIFORNIA INSTITUTE OF TECHNOLOGY & STANFORD UNIVERSITY \\
UNIVERSITY OF CALIFORNIA & UNIVERSITY OF HAWAII \\
MONTANA STATE UNIVERSITY & UNIVERSITY OF TOKYO \\
UNIVERSITY OF NEVADA, RENO & UNIVERSITY OF UTAH \\
NEW MEXICO STATE UNIVERSITY & WASHINGTON STATE UNIVERSITY \\
OREGON STATE UNIVERSITY & UNIVERSITY OF WASHINGTON
\end{tabular}

The Supporting Institutions listed above contribute to the cost of publication of this Journal, but they are not owners or publishers and have no responsibility for its content or policies.

Mathematical papers intended for publication in the Pacific Journal of Mathematics should be in typed form or offset-reproduced (not dittoed), double spaced with large margins. Please do not use built up fractions in the text of the manuscript. However, you may use them in the displayed equations. Underline Greek letters in red, German in green, and script in blue. The first paragraph must be capable of being used separately as a synopsis of the entire paper. In particular it should contain no bibliographic references. Please propose a heading for the odd numbered pages of less than 35 characters. Manuscripts, in triplicate, may be sent to any one of the editors. Please classify according to the 1991 Mathematics Subject Classification scheme which can be found in the December index volumes of Mathematical Reviews. Supply name and address of author to whom proofs should be sent. All other communications should be addressed to the managing editor, or Julie Speckart, University of California, Los Angeles, California 90024-1555.

There are page-charges associated with articles appearing in the Pacific Journal of Mathematics. These charges are expected to be paid by the author's University, Government Agency or Company. If the author or authors do not have access to such Institutional support these charges are waived. Single authors will receive 50 free reprints; joint authors will receive a total of 100 free reprints. Additional copies may be obtained at cost in multiples of 50 .

The Pacific Journal of Mathematics (ISSN 0030-8730) is published monthly except for July and August. Regular subscription rate: $\$ 190.00$ a year (10 issues). Special rate: $\$ 95.00$ a year to individual members of supporting institutions.

Subscriptions, orders for numbers issued in the last three calendar years, and changes of address should be sent to Pacific Journal of Mathematics, P.O. Box 969, Carmel Valley, CA 93924, U.S.A. Old back numbers obtainable from Kraus Periodicals Co., Route 100, Millwood, NY 10546.

The Pacific Journal of Mathematics at P.O. Box 969, Carmel Valley, CA 93924 (ISSN 0030-8730) is published monthly except for July and August. Second-class postage paid at Carmel Valley, California 93924, and additional mailing offices. Postmaster: send address changes to Pacific Journal of Mathematics, P.O. Box 969, Carmel Valley, CA 93924.

PUBLISHED BY PACIFIC JOURNAL OF MATHEMATICS, A NON-PROFIT CORPORATION Copyright $(\mathcal{1} 1993$ by Pacific Journal of Mathematics 


\section{PACIFIC JOURNAL OF MATHEMATICS}

Volume $157 \quad$ No. $2 \quad$ February 1993

Strong integral summability and the Stone-Čech compactification of the half-line

JEFF CONNOR and MARY ANNE SWARDSON

The endlich Baer splitting property

225

THEODORE GERARD FATICONI

The formal group of the Jacobian of an algebraic curve

MARGARET N. FREIJE

Concordances of metrics of positive scalar curvature

PAWEL GAJER

Explicit construction of certain split extensions of number fields and constructing cyclic classfields

STANLEY JOSEPH GURAK

Asymptotically free families of random unitaries in symmetric groups

ALEXANDRU MiHAi NiCA

On purifiable subgroups and the intersection problem

TAKASHI OKUYAMA

On the incidence cycles of a curve: some geometric interpretations

LUCIANA RAMELLA

On some explicit formulas in the theory of Weil representation

R. RANGA RAO

An analytic family of uniformly bounded representations of a free product of 373 discrete groups

JANUSZ WYSOCZAŃSKI

Errata: "Dentability, trees, and Dunford-Pettis operators on $L_{1}$ "

MARIA GIRARDI and ZHIBAO HU

Errata: "Poincaré cobordism exact sequences and characterisation" 